\title{
Maraviroc inhibits SARS-CoV-2 multiplication and s-protein mediated cell fusion in cell culture
}

Kenneth H. Risner ${ }^{1}$, Katie V. Tieu ${ }^{2}$, Yafei Wang ${ }^{3}$, Allison Bakovic ${ }^{1}$, Farhang Alem ${ }^{1}$, Nishank Bhalla $^{1}$, Steven Nathan ${ }^{4}$, Daniel E. Conway ${ }^{2}$, Paul Macklin ${ }^{3}$, and Aarthi Narayanan ${ }^{1}$

${ }^{1}$ National Center for Biodefense and Infectious Diseases, George Mason University, Manassas, Virginia, United States of America

${ }^{2}$ Department of Biomedical Engineering, Virginia Commonwealth University, Richmond, Virginia, United States of America

${ }^{3}$ Intellegent Systems Engineering, Indiana University, Bloomington, Indiana, United States of America

${ }^{4}$ Advanced Lung Disease, Lung Transplant and INOVA Lung Services, INOVA Fairfax Hospital, Fairfax, Virginia, United States of America

Running title: Maraviroc inhibits SARS-CoV-2 multiplication

Correspondence: Dr. Aarthi Narayanan, anaraya1@gmu.edu

Key words: SARS-CoV-2, Maraviroc, FDA approved small molecules and antibodies, viral multiplication, cell fusion, predictive modeling 


\section{Summary}

In an effort to identify therapeutic intervention strategies for the treatment of COVID-19, we have investigated a selection of FDA-approved small molecules and biologics that are commonly used to treat other human diseases. A screen of 19 small molecules and 3 biologics was conducted in cell culture and the impact of treatment on viral titer was quantified by plaque assay. The screen identified 4 FDA-approved small molecules, Maraviroc, FTY720 (Fingolimod), Atorvastatin and Nitazoxanide that were able to inhibit SARS-CoV-2 infection. Confocal microscopy with over expressed S protein demonstrated that Maraviroc reduced the extent of Sprotein mediated cell fusion as observed by fewer multinucleate cells in drug-treated cells. Mathematical modeling of drug-dependent viral multiplication dynamics revealed that prolonged drug treatment will exert an exponential decrease in viral load in a multicellular/tissue environment. Taken together, the data demonstrate that Maraviroc, Fingolimod, Atorvastatin and Nitazoxanide inhibit SARS-CoV-2 in cell culture.

\section{Highlights}

- Maraviroc, FTY720, Nitazoxanide and Atorvastatin inhibit SARS-CoV-2 multiplication in cell culture.

- Maraviroc does not interfere with the interaction between SARS-CoV-2 spike protein and ACE2 receptor.

- Maraviroc exhibits only modest synergistic activities with FTY720, Nitazoxanide or Atorvastatin.

- Maraviroc reduces the extent of SARS-CoV-2 S-protein mediated cell fusion.

- Mathematical modeling reveals that Maraviroc treatment will elicit an exponential decrease in viral load in a multicellular tissue environment. 


\section{Introduction}

Severe Acute Respiratory Syndrome coronavirus 2 (SARS-CoV-2) is a novel coronavirus strain which is the causative agent of the coronavirus disease 2019 (COVID-19) pandemic (Zhou et al., 2020). As of August 2020, there are 18,104,056 confirmed cases of COVID-19 resulting in 689,922 deaths (Johns Hopkins Coronavirus Resource Center, (Dong et al., 2020)). Coronaviruses $(\mathrm{CoV})$ are generally the cause of common colds resulting in mild respiratory infections (Weiss and Leibowitz, 2011).The emergence of pathogenic coronavirus (CoV) strains with high mortality and morbidity has occurred multiple times over the past two decades, (De Wit et al., 2016). Severe acute respiratory virus (SARS) emerged in 2002 (De Wit et al., 2016; Stadler et al., 2003; Zhong and Wong, 2004). Several SARS-like coronaviruses have been identified in bat species (Banerjee et al., 2019; Ge et al., 2013; Menachery et al., 2015; Wang et al., 2006). Middle Eastern respiratory virus was detected in 2012 (De Wit et al., 2016; Mackay and Arden, 2015). In December of 2019, SARS-CoV-2 began circulating in Wuhan, China resulting in the current COVID-19 pandemic. SARS-CoV-2 is known to be transmitted from human-to-human with inhalational acquisition being the primary mode of transfer (Liu and Zhang, 2020; Nishiura et al., 2020; Song et al., 2020).

Coronaviruses belong to the superfamily Nidovirales and subfamily Orthocoronavirinae (Weiss and Leibowitz, 2011). CoVs are enveloped, nonsegmented, positive-sense single-stranded RNA viruses (Weiss and Leibowitz, 2011). They are classified in four sub-groups: alpha, beta, gamma and delta. The seven viruses that are known to infect humans belong to alpha and beta. HCoV229E and HCoV-NL62 are classified as alpha while HCoV-OC43, HCoV-HKU1, MERS-CoV, SARS-CoV, and SARS-CoV-2 are beta CoVs. The virus measures approximately $65-125 \mathrm{~nm}$ in diameter, and the viral genome measures approximately $29.9 \mathrm{~Kb}$ (Astuti and Ysrafil, 2020). The viral genome encompasses 14 open reading frames (ORFs) that encode both structural and nonstructural viral proteins. Among the structural proteins, S protein has achieved significant attention due to the critical role it plays in interaction of the virus with the ACE2 receptor on host cells (Ahmed et al., 2020). In addition to ACE2, the type II transmembrane serine protease (TMPRSS2) is also required for SARS-CoV-2 entry into cells, thus making these two membrane-associated proteins as the primary determinants for viral entry. ACE2 receptor expression can be detected in various organs besides in the lungs, including heart, kidneys and the gastrointestinal tract. Notably, COVID-19 is characterized by disease manifestations that impact all of the ACE2 positive organs and tissues. The critical aspects of interactions between the viral spike protein and the host membrane proteins ACE2 and TMPRSS2 have led to several therapeutic candidates that interfere with this virus-host protein interactions.

Infection by coronaviruses such as infectious bronchitis virus (IBV) is known to result in cellcell fusion and formation of large, multinucleated cells referred to as syncytia (Fehr and Perlman, 2015; Sisk et al., 2018). Newly synthesized S protein either in the context of coronavirus infected cells or cells that over express $\mathrm{S}$ protein, is said to accumulate on the plasma membrane (Lontok et al., 2004).Such S protein enriched sections of plasma membranes can fuse resulting in cell-cell fusion. Inhibition of IBV infected cells with Abl kinase inhibitors (Imatinib) resulted in reduced 
syncytia in addition to decreasing viral load (Sisk et al., 2018). This inhibition of S-protein mediated cell fusion by Imatinib could be achieved even in the absence of other viral proteins suggesting that the cell-cell fusion event in coronavirus infected cells is likely to be dependent only on $\mathrm{S}$ protein function. The cell-cell fusion event mediated by coronavirus $\mathrm{S}$ protein is suggested to be controlled by different host enzymatic components than those that influence virus-host membrane fusion. S-protein dependent cell-cell fusion was shown to be independent of cathepsin L which was essential for virus-cell fusion. A novel leupeptin-sensitive host cell protease activated S protein dependent cell-cell fusion in target cells expressing high levels of ACE2 in the context of SARS-CoV-1 (Simmons et al., 2011).This mechanism of S protein mediated cell-cell fusion was implicated in viral spread in the context of SARS-CoV-1 infection and the ability of the virus to evade host humoral immune responses, thus posing an important challenge for antibody-mediated viral control (Glowacka et al., 2011). Increased incidence of S-protein mediated syncytia were observed in the context of SARS-CoV-2 S-protein, as compared to SARS-CoV-1, thus highlighting the need to address S-protein mediated cell-cell fusion in SARS-CoV-2 to control viral spread in the infected host (Xia et al., 2020).

A significant contributor towards the morbidity and mortality associated with COVID-19 is the host inflammatory response, with several pro-inflammatory cytokines known to be involved in the tissue damage sustained due to infection. Cytokine storm is suggested to be a significant cause of organ failure and Acute Respiratory Distress Syndrome (ARDS) (Ye et al., 2020). Several host signaling events including the NFkB pathway, JAK-STAT pathway and IFN pathway cumulatively contribute to the proinflammatory environment in target tissues such as the lungs. Onset of ARDS during the later stages of COVID-19 is associated with poor prognosis and is a direct outcome of inflammatory lung damage (Fanelli et al., 2013). Additional complications that are unique to COVID-19 include microclots in blood vessels that supply blood to the heart, lungs and the brain (Ciceri et al., 2020). This disseminated coagulation contributes to death resulting from strokes and heart complications in addition to ARDS (Bansal, 2020; Trejo-Gabriel-Galán, 2020).

It is becoming increasingly apparent that COVID-19 is a combined outcome of viral multiplication in multiple target sites in the infected individual and various host-based complications resulting from dysregulated thrombotic and inflammatory events. An effective therapeutic regimen will need to address the viral and host-based aspects of the disease in order to be able to decrease the health burden and increase survival of affected individuals. Furthermore, such an approach may be essential to address any potential long-term complications that may arise in survivors, especially those who are considered to be high-risk populations. High-risk Individuals include those with preexisting health conditions such as coronary heart disease, and inflammatory states including asthma and diabetes. Seeking a therapeutic regimen that may be easily adapted to such high-risk individuals, we focused our attention on a subset of FDA-approved small molecules and biologics that are commonly used to treat heart disease, inflammation and infectious disease. We screened a total of 22 such molecules and have identified four small molecules (Maraviroc, FTY 720, Atorvastatin and Nitazoxanide) and three antibodies (Bevacizumab, Eculizumab and Anti-CD126/IL6R), all of which were able to decrease infectious virus titers in cell culture. Additional studies performed with the prioritized small molecule inhibitors demonstrated that these 
compounds moderately impacted viral entry and interfered with the interaction between the spike protein and ACE2 to varying degrees. Cell biology studies demonstrate that Maraviroc was able to decrease the extent of S-protein-mediated cell fusion. Our efforts to develop simulation models of the antiviral outcomes of Maraviroc on a tissue level demonstrated that sustained availability of drug will have a significant impact on decreasing viral load and increasing cell survival. Simulations involving extending treatment windows, decreasing treatment concentrations and efficacy show a protective effect that may have important implications in clinical settings.

\section{Results}

\section{Identification of anti-SARS-CoV-2 activity of FDA approved small molecules.}

The screen was conducted with 19 FDA-approved small molecules that are commonly prescribed to the general population which included antiviral and anti-parasitic compounds. The screen also included compounds that were documented to alter host signaling cascade events and exhibit anti-inflammatory properties. The list of small molecules that were screened is included in Figure 1A. The screen was conducted using Vero cells and the Washington strain of SARS-CoV-2 (2019-nCoV/USA-WA1/2020). The toxicity of the small molecules in the context of Vero cells was first quantified by treating the cells with the inhibitor at a low, medium and high concentration $(1 \mu \mathrm{M}, 5 \mu \mathrm{M}$ and $10 \mu \mathrm{M})$ for 24 hours after which cell viability was quantified using Cell Titer Glo assay (Figure 1B). Almost all of the small molecules demonstrated less than 10\% cytotoxicity in this assay with the exception of FTY720 and Atorvastatin that showed $20 \%$ and $10 \%$ reduction in cell viability (compared to the untreated control) at the highest concentration. AntiSARS-CoV-2 activities of these small molecules were quantified by plaque assay and the outcomes are included in Figure 1C. Vero cells were pre-incubated with nontoxic concentrations of the compounds for 1 hour prior to infection. Infection with SARS-CoV-2 was carried out by incubating the virus with cells (multiplicity of infection [MOI]: 0.1 ) in the presence of the designated inhibitor for 1 hour. After allowing time for the infection, the virus overlay was removed and media containing the appropriate inhibitor was added back to the cells. DMSO treated cells were maintained as controls alongside. The amount of infectious virus in the supernatants of the drug-treated cells was quantified by plaque assay at 16 hours post infection and compared to the DMSO-treated control.

Among the antiviral compounds that were tested, Maraviroc demonstrated a 30.7-fold decrease $(\mathrm{p}<0.0001)$ in viral load as compared to the DMSO control (Figure 1C). Among the anti-parasitic compounds that were tested, Nitazoxanide demonstrated a 7.1-fold decrease $(p<0.0001)$ when compared to the DMSO control. Hydroxychloroquine was included as an added control and it demonstrated a 26.9-fold decrease $(\mathrm{p}<0.0001)$ is viral titers (Figure 1C). Interestingly, Nitazoxanide was suggested as a potential anti-SARS-CoV-2 therapeutic based on prior activity observed against MERS-CoV (Martins-Filho et al., 2020). Nitazoxanide was also suggested as a combinatorial therapeutic along with Azithromycin (Kelleni, 2020). Nitazoxanide is currently undergoing clinical trials for safety and efficacy to treat COVID-19 patients (ClinicalTrials.gov Identifier: NCT04435314). In the set of compounds that influenced host signaling and inflammatory events, 
FTY-720 showed a 38.4-fold decrease $(\mathrm{p}<0.0001)$ of SARS-CoV-2 viral titer as compared to the DMSO control. FTY-720 is FDA-approved for the treatment of multiple sclerosis and is documented to exhibit anti-inflammatory activities by modulating the NFkB signaling cascade (Pul et al., 2016). In this class of compounds, Atorvastatin, a commonly used statin for the treatment of high cholesterol, was noted to decrease viral load 1.6-fold $(\mathrm{p}<0.01)$ as compared to the DMSO control. The relative percent inhibitions of these prioritized compounds is indicated in Figure 1D, in comparison with the DMSO control.

\section{Identification of anti-SARS-CoV-2 activity of FDA approved biologics.}

Three FDA-approved biologics namely, Bevacizumab, Eculizumab and CD126/IL6R mAb were evaluated for anti-SARS-CoV-2 activity in a similar manner as described above for small molecules (Figure 2A). Bevacizumab (Avastin) is an inhibitor of VEGF-R2 by binding to VEGF-A and is used for the treatment of several types of cancers (Mahfouz et al., 2017; Papachristos et al., 2019). Eculizumab (Soliris) is undergoing clinical trials for use as a therapeutic for the treatment of COVID-19 patients, potentially for the anti-inflammatory activities and the ability to moderate inflammation related complications (ClinicalTrials.gov Identifier: NCT04288713). Briefly, toxicity of the biologics was evaluated by incubating Vero cells with a low $(1 \mu \mathrm{g})$ and high $(2.5 \mu \mathrm{g})$ concentration of the target biologic for 24 hours after which cell viability was quantified by Cell Titer Glo (Figure 2B). Less than 10\% cytotoxicity was detected for these molecules at the concentrations tested, with the exception of CD126/IL-6R antibody. A concentration of $2.5 \mu \mathrm{g}$ was selected for efficacy evaluation for the less toxic biologics and $1 \mu \mathrm{g}$ was selected for the CD126/IL-6R antibody. Impact of inhibitor treatment on SARS-CoV-2 infectivity was assessed by performing infection of Vero cells (MOI: 0.1 ) in the presence of the biologic at nontoxic concentration for 1 hour. The infected cells were incubated with the biologic for an additional 16 hours after which infectious virus load in the supernatant was quantified in comparison with the DMSO control (Figure 2C). Bevacizumab and Eculizumab treatment resulted in a 4.2-fold decrease $(p<0.0001)$ and a 3.7-fold decrease of virus titers, respectively. CD126/IL6R antibody resulted in 1.9 -fold decrease of virus load.

\section{Impact of small molecule inhibitors on early steps of viral infection.}

The experimental design for the assessment of anti-SARS-CoV-2 activities of the small molecule inhibitors as included in Figure 1C involved performing the infection step in the presence of the inhibitor. The extent of inhibition observed due to the presence of the drug during the infection step may suggest a potential impact of the drug on the early steps of infection. To ascertain if there was any impact of the four prioritized small molecules on early steps of infection, the experiment was performed without including the drugs during the infection step. Vero cells were pretreated with Maraviroc, FTY-720, Nitazoxanide and Atorvastatin for 1 hour after which the inhibitor-containing medium was removed. Virus-containing inoculum was added to the cells without including the inhibitor and infection was allowed to progress for 1 hour, after which the virus inoculum was removed. Fresh media with the inhibitors were added back to the cells and viral load in the supernatants was quantified by plaque assay at 16 hours post infection (Figure 3). The extent of inhibition observed with Maraviroc, Fingolimod and Nitazoxanide was reduced 
to 3.9-fold $(\mathrm{p}<0.0001), 5.3$-fold $(\mathrm{p}<0.0001)$ and 6.7-fold $(\mathrm{p}<0.0001)$ respectively. Both Maraviroc and FTY720 were 7-times less effective when absent during the viral inoculation, whereas Nitazoxanide performed almost as well regardless of its presence during the time of infection, suggesting that these drugs interfered with some aspect of the early infection and viral introduction process. Atorvastatin treatment reduced titers 1.4 -fold but was not statistically significant.

\section{Interference of anti-SARS-CoV-2 small molecules with ACE2:Spike protein interaction.}

The observation that Maraviroc, FTY720 and Nitazoxanide increased the extent of inhibition when drug was included at the time of infection suggested that the drugs may exert an effect on the interaction of the viral surface proteins with the cellular receptor target. To that end, a VSV$\mathrm{G}$ pseudovirus with a firefly luciferase reporter which retains the SARS-CoV-2 spike protein on the surface was used to query if the prioritized compounds affected early steps of the infectious process. The pseudovirus is capable of ACE2-based viral entry which can be quantified by measuring intracellular luciferase expression. As the pseudovirus is not capable of viral RNA synthesis, the inhibitory effect is exclusive to the early entry and post entry steps. The pseudovirus was used to infect Vero cells in the presence of the inhibitor and luciferase expression was measured at 16 hours post infection (Figure 4A). While Nitazoxanide exerted a 74.1\% inhibition of luciferase expression, Maraviroc and FTY720 produced $28.6 \%$ and a $41.1 \%$ inhibition, respectively, of reporter expression suggesting that Maraviroc or FTY720 were likely to inhibit additional steps besides entry to achieve viral inhibition.

To further ascertain if this observed inhibition to viral entry exerted by Nitazoxanide involved interference in the molecular interaction between the viral spike protein and the cellular ACE2 receptor, a biotinylated receptor binding assay was employed. In this assay, the potential for the inhibitor to interfere with the binding of spike protein and ACE2 receptor is quantified as a measure of biotin expression. The assay involves using a nickel coated plate to which his-tagged ACE2 is added. The drug is then added to the plate and incubated for 1 hour at room temperature after which the SARS-CoV-2 spike protein bound to biotin is added and the plate is incubated for 1 hour after which Streptavidin-HRP is added. Chemiluminescence is measured following the addition of HRP substrate which is a reflection of the interaction between the ACE2 protein and S protein. Quantification of luminescence in drug-treated wells as compared to the DMSOtreated control demonstrated that Maraviroc, FTY 720 or Nitazoxanide did not interfere with the interaction at lower concentration of the inhibitor (Figure 4B). Atorvastatin elicited a modest interference in the interaction as demonstrated by a reduction of $31.7 \%(\mathrm{p}<0.01)$ of biotin expression (Figure 4B). When the experiment was conducted at higher concentrations of the drug (100 $\mu \mathrm{M})$, FTY720 was able to exert a modest interference $(27 \%$ relative to control, $\mathrm{p}<0.01)$, while Maraviroc and Nitazoxanide treatment did not produce any impact on the interaction (Figure 4C). The data suggest that the mechanism of inhibition for Maraviroc and Nitazoxanide is unlikely to involve disruption of receptor binding by the viral spike protein and is likely that early post-entry steps are impacted by the drug treatment.

\section{Impact of Maraviroc on intracellular events in SARS-CoV-2 infected cells.}


The observations that Maraviroc exhibited strong inhibition of SARS-CoV-2 when infection was performed in the presence of the inhibitor, without necessarily impacting the interaction of the viral surface spike protein with the ACE2 receptor suggested that alternate mechanisms that may be critical for the establishment of a productive viral infection may be impacted by Maraviroc. Evidence in the literature indicates that the coronavirus spike protein drives membrane fusion resulting in the formation of multinucleate cells (Fehr and Perlman, 2015; Lontok et al., 2004; Sisk et al., 2018). Notably, this phenomenon was observed to be elicited by over expressed S-protein (spike protein) as well, suggesting that it is independent of functionality from other proteins. As a next step, the impact of Maraviroc on S-protein based cellular fusion was assessed by confocal microscopy (Figure 5). Vero cells were transfected with an over expression construct that expressed the SARS-CoV-2 spike protein. Transfected cells were maintained in the presence of Maraviroc for up to 48 hours to permit expression of S-protein. DMSO-treated cells were maintained as controls to ascertain baseline numbers of multinucleate cells. The cells were fixed and stained at 48 hours post transfection and the extent of formation of multinucleate cells were quantified by confocal microscopy (Figure 5A). The data demonstrate that increased expression of S-protein in Vero cells results in the formation of large multinucleate cells that typically contain 4-8 nuclei per cell. Vero cells transfected with a GFP control plasmid did not form multinucleate cells (data not shown). Interestingly, maintaining the transfected cells in the presence of Maraviroc reduced the frequency of multinucleate cell formation by approximately 2-fold as compared to the DMSO-treated controls (Figure 5B), as well as decreasing the average number of nuclei in these clusters (Figure 5C). This outcome suggests the possibility that Maraviroc treatment may result in a decrease in viral load in the cell population by preventing membrane fusion events that impact intracellular viral multiplication and spread.

\section{Determination of synergy between the anti-SARS-CoV-2 small molecules.}

The ability of Maraviroc to exert synergistic inhibitory outcomes when combined with FTY720, Atorvastatin or Nitazoxanide was assessed. As a first step, toxicity of the combination of drugs was assessed when the drugs were combined at $20 \%, 50 \%$ and $100 \%$ of their effective concentrations as observed in Figure 1D. To that end, the four compounds were assessed when they were combined at $1 \mu \mathrm{M}$ each, $2.5 \mu \mathrm{M}$ each and $5 \mu \mathrm{M}$ each. The combinations were used to treat Vero cells and cell viability was quantified at 24 hours post treatment by Cell Titer Glo assay (Figure $6 \mathrm{~A}$ ). The combinations that resulted in $>90 \%$ cell survival ( $<10 \%$ toxicity) were considered for efficacy assessment. To assess potential synergy between these compounds, infection with SARS-CoV-2 were carried out in the presence of the drug combinations and the infected cells were maintained for 16 hours in the presence of the combinations. The viral load in the supernatants were quantified at 16 hours post infection by plaque assay and relative inhibition compared to that of the DMSO control (Figure 6B). A modest increase in inhibitory potential was noted when Maraviroc was combined with any of the three compounds (Figure 6C). Inhibitory potential was increased 2.6\% for FTY720, 10.6\% for Atorvastatin and 11.9\% for Nitazoxanide.

Remdesivir was also screened for antiviral activity and possible synergy with Maraviroc. Toxicity of Remdesivir alone was below $10 \%$ for the concentrations that were screened. Combining 
Remdesivir with Maraviroc did not substantially increase cytotoxicity, and cell viability remained above $90 \%$. Remdesivir alone decreased viral titer by $91.1 \%(\mathrm{p}<0.0001)$. When Maraviroc was added to the treatment viral titers were decreased by $94 \%(p<0.0001)$.

\section{Modeling of Maraviroc mediated inhibition of SARS-CoV-2 in a multicellular environ- ment.}

To study the dynamics of SARS-CoV-2 spread under control and Maraviroc-inhibited conditions, we adapted a computational model of SARS-CoV-2 (Getz et al., 2020) under the assumption that the drug inhibits viral endocytosis and exocytosis, but does not inhibit ACE2 receptor binding. We tuned the viral binding, endocytosis, replication, release and pharmacodynamics parameters to match the viral titer at 16 hours under no treatment and $5 \mu \mathrm{M}$ of Maraviroc. The fitted model suggests that exocytosis is not fully inhibited at $5 \mu \mathrm{M}$ : because the original 3000 virions are quickly bound and endocytosed, replication and exocytosis are required to explain the presence of unbound virus at 16 hours. Moreover, because Maraviroc does not inhibit SARSCoV-2 binding to ACE2 receptors on uninfected cells, sustained replication and exocytosis are required to outpace binding to ACE2 receptors. Despite of incomplete inhibition, fewer cells are infected by 16 hours, and fewer infected cells have a high viral load of assembled virions. (Supplemental Figure S1.)

When we extended the simulations to 2 days (Figure 7A), we found that the $5 \mu \mathrm{M}$ dose delayed but did not prevent the spread of the infection. By 2 days, all viable cells were infected with high viral load (bright yellow cells), but cell survival was increased over the untreated control. Increasing the dose to $10 \mu \mathrm{M}$ further increased cell survival, and some infected cells still remained at 2 days. Plots of the uninfected, infected, and dead cell counts over 5 days also showed increasing delays as the drug dose was increased (bottom plots in Figure 7A). Viral titer dropped significantly as dose increased, while viable cell fraction improved (Figure 7C). Results for other doses are plotted in Supplementary Figure S2.

Next, we investigated whether Maraviroc (at $10 \mu \mathrm{M}$ ) could still slow an infection if administered after the initial infection. For shorter delays between infection and dosing ( 0 to 8 hours), there was still a substantial increase in cell survival and reduction in viral titer at 48 hours (Figure 7B, 7D), which delays over 8 hours lead to infection dynamics that closely mirrored the untreated control. However, even a 24-hour delay in dosing still reduced viral titer by at least a factor of 2 by 48 hours compared to the untreated control. (Further delays are plotted in Supplementary Figure S4). We also found if the maximum sensitivity to the drug (i.e., the maximum inhibition of exocytosis and endocytosis) was reduced from $100 \%$ to $90 \%$, there was still a substantial antiviral effect, although the impact of the drug dropped rapidly as sensitivity was further reduced (Supplementary Figure S3.)

\section{Discussion}

SARS-CoV-2, the causative agent of the ongoing COVID-19 pandemic, continues to have a devastating effect globally, increasing the morbidity and mortality burden on a daily basis. The disease appears to have a high probability of severe outcomes in aged populations and those with pre-existing health conditions including cardiovascular disease, asthma and immune disorders. 
From the perspective of bringing quick treatment options to the clinic, there continues to be heightened focus on repurposing FDA-approved drugs. When a therapeutic intervention approach to treat COVID-19 is to be evaluated, it cannot be ignored that the population that presents itself at the clinic is likely to fall in the more severe disease scale, with a high probability of co-morbidities and ongoing medication regimens. This possibility has to be considered in two angles: 1. the aggressive therapeutic strategy that may be used to treat SARS-CoV-2 infection should not interfere with ongoing drug regimens or exert unforeseen catastrophic outcomes; 2 . the possibility of treatment strategies that are commonly used to treat disease conditions such as cardiovascular disease, asthma or immune system disorders may be used to also treat SARSCoV-2 infection. The latter aspect offers not only the possibility of being able to safely use some of these therapeutics in the treatment of these high risk groups, but also paves the way for determining if any of these ongoing therapeutic regimens may offer a level of protection to the target group by preventing escalation of disease severity.

In this study, a total of 22 small molecules and biologics that are commonly used to treat disease states including viral, bacterial and parasitic infections, heart disease and inflammatory conditions such as multiple sclerosis were analyzed for potential antiviral activity against SARS-CoV2 in a cell culture model. The screen identified four small molecules that demonstrated statistically significant anti-SARS-CoV-2 activity, namely, Maraviroc, FTY720, Nitazoxanide and Atorvastatin. Maraviroc elicited a statistically significant and consistent inhibitory effect against SARS-CoV-2 (Figure 1C). Maraviroc is an inhibitor of the cellular CCR5 receptor and is used to treat HIV infection. Interestingly, Maraviroc is under consideration for a clinical trial as a COVID-19 therapeutic as it is known to be well tolerated in human populations, even in the context of a compromised immune system (ClinicalTrials.gov Identifier: NCT04435522). The rationale behind the use of Maraviroc is that it would be beneficial in preventing immune cell depletion (or exhaustion) and increasing routing of inflammatory cells away from sites that sustain inflammatory damage during COVID-19 disease, thus additively contributing to lowering viral load and inflammatory damage in organs such as the lungs. In a small subset of patients who were treated with Maraviroc during the early stages of infection, positive outcomes were noted including decreased onset of clinical parameters (Dr. Steven Nathan, personal communication).

FTY720 (Fingolimod) is a drug that is used to treat multiple sclerosis which demonstrated robust inhibitory activity against SARS-CoV-2 (Figure 1C). It functions as an immune modulator that is currently undergoing clinical trials for use as a COVID-19 therapeutic (ClinicalTrials.gov Identifier: NCT04280588). The rationale for consideration of FTY720 is that it may be able to reduce the inflammatory manifestations of ARDS in the lung by decreasing pulmonary edema and hyaline membrane formation, possibly if used in conjunction with additional methods including ventilator support. Furthermore, the mechanisms of FTY720-mediated reduction of egress of autoreactive lymphocytes from lymphoid tissues may play additional roles in decreasing systemic inflammatory damage. FTY720 treatment may produce short term fluctuations in cardiovascular parameters and blood pressure due to agonistic and antagonistic impacts on Sphingosine-1-phosphate receptor (S1PR) which is differentially expressed in tissues including cardiac cells (Camm et al., 2014). FTY720 has also been implicated in cases of Fingolimod associated macular edema, especially when administered in the same regimen as for multiple sclerosis (Pul et al., 
2016). These factors will require consideration if FTY720 is to be evaluated for COVID-19 treatment.

Nitazoxanide, an FDA-approved drug used to treat diarrhea, exerted antiviral activity in the context of SARS-CoV-2 infection (Figure 1C). There have been recent published reports that refer to the use of Nitazoxanide in combination with other drugs including Azithromycin and Ivermectin, both of which were demonstrated to show anti-SARS-CoV-2 activity in cell culture (Kelleni, 2020). Nitazoxanide has been demonstrated to have antiviral activity against MERS-CoV and potentiate interferon responses (Rossignol, 2016). In the context of MERS-CoV infection model, Nitazoxanide was also shown to decrease the expression of the proinflammatory cytokine IL6, which is also implicated in the inflammatory damage elicited due to SARS-CoV-2 infection. Nitazoxanide is under consideration for a clinical trial as a COVID-19 treatment strategy (ClinicalTrials.gov Identifier: NCT04435314).

Atorvastatin, a commonly used small molecule for the treatment of cardiovascular disease demonstrated modest antiviral activity against SARS-CoV-2 when compared to the other effective compounds (Figure 1C). Recently published outcomes suggested that statin use in patients is likely to be linked to decreased mortality, ventilator use and hospitalizations in the case of COVID-19 patients (Zhang et al., 2020). Atorvastatin is currently undergoing clinical trials as adjunctive therapy for potentially addressing the cardiovascular complications associated with COVID-19 (ClinicalTrials.gov Identifier: NCT04380402).

Studies performed by varying the infection condition, specifically the presence or absence of the candidate drug at the time of infection revealed that including the drug during infection produced a higher rate of inhibition (Figure 3). This observation lead to the hypothesis that the candidate compounds, Maraviroc, FTY720, Nitazoxanide and Atorvastatin may interfere with entry and/or early post-entry steps in the SARS-CoV-2 infectious process. To investigate this possibility further, a VSV-G pseudovirus which expresses the SARS-CoV-2 spike protein on the surface was used in the context of these inhibitors. The extent of inhibition observed with the pseudovirus was significantly lower than what was noted in the context of SARS-CoV-2 (Figure 1C versus Figure 4A) suggesting that additional steps beyond viral entry are likely to be influenced by these small molecules. It may also be possible that the VSV glycoproteins that are also expressed on the surface of the pseudovirion may interfere with the inhibition that these compounds may exert on the SARS-CoV-2 spike protein. Nevertheless, the observation that the early entry steps are impacted by these compounds led to the question whether they interfered with the interaction between the viral spike protein and the host ACE2 receptor. Analysis of binding interaction by a reporter assay in the presence and absence of the inhibitor revealed that while Atorvastatin may elicit a modest level of interference in the interaction between the spike protein and ACE2, Maraviroc and FTY720 did not, even at higher concentrations (Figure 4B). Maraviroc and FTY720 were the most potent of the inhibitors that were tested in this effort and the data supported the idea that the mechanism of inhibition is not likely to involve interference with receptor binding.

Coronavirus spike protein has been shown to be involved in membrane fusion, both in the context of fusion between viral and host cell membranes and in the context of host cell membranes 
(Tang et al., 2020). The membrane fusion event is believed to be mediated by the S2 domain of the extracellular stalk portion of the spike protein. Within the S2 domain, a short, loosely defined fusion peptide, a stretch of 15-25 amino acids is important to initiate the fusion event and is considered to be very sensitive to mutations (Madu et al., 2009). The SARS-CoV-2 fusion peptide region in S2 is considered to be $93 \%$ similar to that observed in SARS-CoV-1, thus indicating a high degree of conservation in the fusion peptide (Tang et al., 2020). SARS-CoV-1 S protein was shown to initiate cell fusion between human cells, which was also observed in the case of SARSCoV-2 (Ou et al., 2020) and the protease TRPRSS11a is thought to aid this fusion event because of S protein processing in the context of SARS-CoV-1 (Kam et al., 2009). Studies that were conducted with an over expressed SARS-CoV-2 S protein expression construct demonstrated an increased incidence of multinucleate cells with an average of 4-7 nucleic per multinucleate cell (Figure 5). When the transfected cells were maintained in the presence of Maraviroc, a statistically significant reduction in the number of multinucleate cells were observed (Figure 5B). This observation raises the interesting possibility of viral spread in a multicellular environment mediated by cell fusion. Such a possibility holds significant relevance to the efficacy of convalescent serum use as an intervention strategy because the intracellular viral population will be shielded from the antibodies and will continue to persist. Synthetic antibodies or nanobodies that are membrane permeable may prove to be effective in such cases. If such a possibility is true, it will explain the observed cases of "relapse" or "prolonged persistence" of viral RNA even in individuals who do not exhibit any disease symptoms. Such an event will also have a significant impact on vaccine design, particularly those that rely on humoral responses to extracellular virus, drawing attention to CD8 mediated responses as being an important requirement for an efficacious vaccine.

An interdisciplinary coalition is actively developing an open source, multiscale simulation model for SARS-CoV-2 infection dynamics in tissue (Getz et al., 2020), which can model the heterogeneous dynamics of individual cells and the spread of viral particles through a tissue (or cell culture). This study adapted and extended the simulator by adding a Maraviroc pharmacodynamics model to each cell and calibrating to the untreated control and $5 \mu \mathrm{M}$ experiments at 16 hours. As employed here, the simulator emphasizes the early entry and post entry events in the context of Maraviroc treatment as a test case with a goal of implementing a full in vivo simulation model to assess the clinical applicability of the drug. The simulations revealed that the inhibition is likely to extend protective effects in a multicellular setting and decrease cell death in relation to a lowered viral load if the effective drug concentration can be maintained. Because the model explicitly simulates viral binding, endocytosis, replication, release and spread, it can help "fill in the gaps" between limited experimental observations. In this effort, the model suggests that antiviral drugs such as Maraviroc can reduce viral titer by nearly two orders of magnitude even with partial inhibition of its target. It also suggests that differences in cell viability may not manifest at early time points, even when a drug completely inhibits viral endocytosis and exocytosis. Moreover, it also suggests that Maraviroc treatments may still be beneficial even when treatment is delayed (e.g., due to delays in diagnosis). Future simulation studies will assess whether Maraviroc can act synergistically with innate immune responses to improve control of the virus and reduce tissue damage. While simulations were employed in the context of Maraviroc as an initial 
demonstration of utility, the additional inhibitors identified in this study will be queried independently for control of viral and inflammatory load, relative pharmacodynamics properties as related to antiviral and anti-inflammatory activities and synergistic outcomes. The simulation model could also afford the opportunity to assess the relative impact of cell-to-cell viral transmission (e.g., via fusion) compared to diffusive dissemination of endocytosed virus particles, and whether treatments like Maraviroc could ultimately halt these alternative forms of viral spread in tissues.

In conclusion, our data demonstrate that Maraviroc, FTY720, Nitazoxanide and Atorvastatin are viable candidates that may be used to treat individuals infected with SARS-CoV-2. These drugs are commonly used to treat a wide demographic of the human population and are known to be well tolerated in humans. The data presented in this effort will also provide support to the ongoing clinical trials that are exploring the utility of these compounds as COVID-19 treatment strategies.

\section{Acknowledgements.}

The following reagent was deposited by the Centers for Disease Control and Prevention and obtained through BEI Resources, NIAID, NIH: SARS-Related Coronavirus 2, Isolate USAWA1/2020, NR-52281. We would like to thank Dr. Rick Holtsberg for kindly providing us the VSV-G pseudovirus. This research was supported by GMU start up funds to AN; NIH R35GM119617, NSF CMMI-1653299, and VCU VCU COVID-19 Rapid Research Funding program to DC; , and by generous support from the Jayne Koskinas Ted Giovanis Foundation for Health and Policy (PM and YW). YW and PM acknowledge access to FutureSystems at the Digital Science Center, Luddy School of Informatics, Computing, and Engineering, and to the Big Red 3 supercomputer at Indiana University, Bloomington. We would like to thank all members of the Narayanan laboratory for help with manuscript review.

\section{Author Contributions}

Conceptualization, K.R., K.T., Y.W., S.N., D.C., P.M. and A.N.; Methodology, K.R., K.T., Y.W., D.C., P.M. and A.N.; Software, Y.W. and P.M.; Formal Analysis, K.R., K.T. and Y.W.; Investigation, K.R., K.T., Y.W., A.B., F.A. and N.B.; Resources, K.R., K.T., Y.W., A.B., F.A., N.B., S.N., D.C., P.M. and A.N; Writing - Original Draft, K.R., K.T., Y.W., S.N., D.C., P.M. and A.N.; Writing - Review \& Editing, K.R., K.T., Y.W., A.B., F.A., N.B., S.N., D.C., P.M. and A.N.; Visualization, K.R., K.T., and Y.W.; Supervision, A.N., D.C. and P.M.; Funding Acquisition, A.N., D.C., P.M. and S.N.

\section{Declaration of Interests}

The authors declare no conflict of interest with any aspect of this work. 


\section{Figure Legends}

\section{Figure 1 - Efficacy of small molecule inhibitors against SARS-CoV-2 in Vero cells 16 hpi} with inhibitor in viral inoculum.

(A.) Classification of the Small molecule inhibitors that were tested.

(B.) Drug toxicity in Vero cells at 24 hours. Cells were seeded at a density of 3E4 and overlaid with media conditioned with $0.1 \%$ DMSO or the indicated concentration of inhibitor. Cells were incubated for 24 hours at $37^{\circ} \mathrm{C}$ at $5 \% \mathrm{CO} 2$. Viability was determined using CellTiter Glo assay.

(C.) Efficacy of small molecule inhibitors in Vero cells 16 hpi. Cells were seeded at a density of $3 \mathrm{E} 4$ and overlaid with conditioned media containing $0.1 \%$ DMSO or $5 \mu \mathrm{M}$ of inhibitor. Cells were incubated for 2 hours. Conditioned media was removed. Viral inoculum (MOI: 0.1) containing $5 \mu \mathrm{M}$ of inhibitor was added to cells and incubated for 1 hour at $37^{\circ} \mathrm{C}$ and $5 \% \mathrm{CO} 2$. Viral inoculum was removed, cells were washed with DPBS, and conditioned media was returned to the cells. Culture was incubated for 16 hpi. Supernatants were collected and a viral plaque assay was used to quantify the viral titers.

(D.) Inhibition that was observed at $16 \mathrm{hpi}$, represented as relative percentages.

The quantitative data are depicted as the mean of three biologically independent experiments \pm SD. $* p<0.05 ; * * p<0.01 ; * * * p<0.001 ; * * * * p<0.0001$.

Figure 2 - Efficacy of antibodies against SARS-CoV-2 in Vero cells 16 hpi.

(A.) List of antibodies that were screened.

(B.) Antibody toxicity in Vero cells at 24 hours. Cells were seeded at a density of 3E4 and overlaid with media conditioned with $0.1 \% \mathrm{DMSO}$ or the indicated concentration of antibody. Cells were incubated for 24 hours at $37^{\circ} \mathrm{C}$ at $5 \% \mathrm{CO} 2$. Viability was determined using CellTiter Glo assay.

(C.) Efficacy of antibodies in Vero cells 16 hpi. Cells were seeded at a density of 3E4 and overlaid with conditioned media containing $0.1 \%$ DMSO or maximum permissible dose of antibody. Cells were incubated for 2 hours. Conditioned media was removed. Viral inoculum (MOI: 0.1) was added to cells and incubated for 1 hour at $37^{\circ} \mathrm{C}$ and $5 \% \mathrm{CO} 2$. Viral inoculum was removed, cells were washed with DPBS, and conditioned media was returned to the cells. Culture was incubated for $16 \mathrm{hpi}$. Supernatants were collected and a viral plaque assay was used to quantify the viral titers.

(D.) Inhibition that was observed at $16 \mathrm{hpi}$, represented as relative percentages.

The quantitative data are depicted as the mean of three biologically independent experiments \pm SD. $* p<0.05 ; * * p<0.01 ; * * * p<0.001 ; * * * * p<0.0001$. 
Figure 3 - Efficacy of small molecule inhibitors against SARS-CoV-2 in Vero cells 16 hpi without inhibitor in viral inoculum.

(A.) Cells were seeded at a density of $3 \mathrm{E} 4$ and overlaid with conditioned media containing $0.1 \%$ DMSO or $5 \mu \mathrm{M}$ of inhibitor. Cells were incubated for 2 hours after which conditioned media was removed. Viral inoculum (MOI: 0.1) containing no inhibitor was added to cells and incubated for 1 hour at $37^{\circ} \mathrm{C}$ and $5 \% \mathrm{CO}$. Viral inoculum was removed, cells were washed with DPBS, and conditioned media was returned to the cells after which cells were cultured for 16 hpi. Supernatants were collected and plaque assay was used to quantify the viral titers.

(B.) Inhibition that was observed at $16 \mathrm{hpi}$, represented as relative percentages.

The quantitative data are depicted as the mean of three biologically independent experiments \pm SD. ${ }^{*} p<0.05 ; * * p<0.01 ; * * * p<0.001 ; * * * * p<0.0001$.

Figure 4 - rVSV Pseudotyped SARS-CoV2 Spike Neutralization Assay and ACE2: Spike S1Biotin (SARS-CoV-2) Inhibitor Screening Assay.

(A.) Luminescence was measured to determine extent of neutralization compared to $0.1 \%$ DMSO control. Vero cells were seeded at a density of 6E6. rVSV virus was incubated with indicated drug at a final concentration of $5 \mu \mathrm{M}$ or $0.1 \%$ DMSO for one hour. Media was removed from plate, replaced with treated or untreated virus. Plate was incubated for one hour at $37^{\circ} \mathrm{C}$ and $5 \% \mathrm{CO} 2$. Conditioned media added to plate and incubated overnight. Cells were lysed and Firefly Luciferase was added to the plate and luminescence immediately measured.

(B.) Assay plate was coated with his-tagged. Inhibitor was added to bring final concentration to indicated amount. Plate was incubated for 1 hour at room temperature. SARS-CoV-2 spike S1biotin was added and incubated for 1 hour. Streptavidin-HRP was added and incubated for 1 hour. HRP substrate was added and chemiluminescence was immediately measured.

The quantitative data are depicted as the mean of three biologically independent experiments \pm SD. $* p<0.05 ; * * p<0.01 ; * * * p<0.001 ; * * * * p<0.0001$. 
Figure 5 - Maraviroc inhibits cell-cell fusion.

(A.) Vero cells were transfected with S protein tagged with Strep-tag II and treated with DMSO (labeled as condition ND for no drug) or $5 \mu \mathrm{M}$ Maraviroc for 48 hours (treated immediately after transfection; labeled as condition A), or $5 \mu \mathrm{M}$ Maraviroc for 72 hours (treated 24 hours before transfection, labeled as condition B). Immunohistochemistry showed that $\mathrm{S}$ protein expression resulted in the formation of multinucleated cells, which was inhibited with Maraviroc treatment.

(B.) The frequency of multinucleated cells, normalized to total cell number, was reduced with Maraviroc treatment.

(C.) The average number of nuclei in multinucleated cells was also reduced with Maraviroc treatment.

The quantitative data are depicted as the mean of three biologically independent experiments \pm Standard Error ** $p<0.01 ; * * * p<0.001$

Figure 6 - Synergistic effects of drug combinations against SARS-CoV-2 in Vero cells at 16 hpi.

(A.) Combination drug toxicity in Vero cells at 24 hours. Cells were seeded at a density of 3E4 and overlaid with media conditioned with $0.1 \% \mathrm{DMSO}$ or the indicated concentration of inhibitor. Cells were incubated for 24 hours at $37^{\circ} \mathrm{C}$ at $5 \% \mathrm{CO}$. Viability was determined using CellTiter Glo assay.

(B.) Efficacy of inhibitors combinations in Vero cells at 16 hpi. Cells were seeded at a density of 3E4 and overlaid with conditioned media containing 0.1\% DMSO or 5:5 $\mu \mathrm{M}$ of Maraviroc plus indicated inhibitor. Cells were incubated for 2 hours and conditioned media was removed. Viral inoculum (MOI: 0.1 ) containing 5:5 $\mu \mathrm{M}$ of inhibitor combination was added to cells and incubated for 1 hour at $37^{\circ} \mathrm{C}$ and $5 \% \mathrm{CO} 2$. Viral inoculum was removed, cells were washed with DPBS, and conditioned media was returned to the cells. Culture was incubated for $16 \mathrm{hpi}$ after which supernatants were collected and a viral plaque assay was used to quantify the viral titers.

(C.) Inhibition that was observed at $16 \mathrm{hpi}$, represented as relative percentages.

(D.) Cytotoxicity of Remdesivir alone and in combination with Maraviroc.

(E.) Efficacy of Remdesivir alone and in combination with Maraviroc in Vero cells at 16 hpi.

The quantitative data are depicted as the mean of three biologically independent experiments \pm SD. ${ }^{*} p<0.05 ; * * p<0.01 ; * * * p<0.001 ; * * * * p<0.0001$. 
Figure 7 - Maraviroc simulation results.

(A.) Simulated infected cell populations (MOI: 0.1 ) at $0,5 \mu \mathrm{M}$, and $10 \mu \mathrm{M}$ of Maraviroc, plotted in the top row at 48 hours. Cells are shaded by viral load, from deep blue (uninfected) to bright yellow (high viral load). Black cells are dead, and white regions represented cleared cells after death. The bottom row plots the dynamics of uninfected cells, infected cells, and dead cells over 5 days. (Mean values for $n=10$ simulations for each condition), demonstrating how increasing doses slow the infection's dynamics.

(B.) Simulated cell populations (MOI: 0.1) after a delayed $10 \mu \mathrm{M}$ Maraviroc dosing, with delays increasing from 0 to 24 hours. Increasing treatment delays reduce the antiviral effects, but even a 24-hour delay increases cell survival and reduces viral titer compared to the untreated control.

(C.) Surviving cell counts (green) at 48 hours increase with dose, while the viral titer (blue) decreases; even the incomplete inhibition at $5 \mu \mathrm{M}$ improves cell survival and reduced titer at 48 hours.

(D.) Surviving cell counts (green) decrease and viral titer (blue) increases as a $10 \mu \mathrm{M}$ dose is delayed from 0 to 24 hours. However, even a 24-hour delay still yields a reduced viral titer and increased cell survival compared to the untreated control.

\section{Materials and Methods}

\section{Virus, Cell lines and reagents.}

SARS-CoV-2 (2019-nCoV/USA-WA1/2020) was obtained from BEI Resources (NR-52281). VERO African green monkey kidney cells (ATCC Cat\# CCL-81) were obtained from the American Type Culture Collection. African green monkey kidney cells (Vero) were maintained in DMEM supplemented with 5\% heat-inactivated fetal bovine essence (FBE) (VWR Life Sciences 10803-034), 1\% Penicillin/Streptomycin, and 1\% L-Glutamine at $37{ }^{\circ} \mathrm{C}$ and $5 \% \mathrm{CO} 2$. All reagents for cell maintenance were pre-warmed to $37^{\circ} \mathrm{C}$ before use.

\section{Inhibitors and Antibodies}

Antibodies Eculizumab (HCA312), Bevacizumab (HCA182) and CD126/IL-6R (AHP2449) were obtained from Biorad.

Dipyridamole (06-915-00), Ciclesonide (63-575), FTY720 (61-761-0), Pirfenidone (P1871100MG), Atorvastatin (A24761G), Indomethacin (1065525G), Bromhexine HCl (B405425G), Maraviroc (37-561-0), Darunavir (67-101-0), Danoprevir (50-190-4862), Azithromycin (37-715-0), Nitazoxanide (N1031200MG) and Tiecoplanin (AC455400010) were obtained from ThermoFisher Scientific. 
Valganciclovir (S4050), Rimantadine (S5484), Letermovir (S8873), Baloxavir (S5952), Hydroxychloroquine Sulfate (S4430) were obtained from Selleckchem.

\section{Viral infection and inhibitor treatment.}

Vero cells were seeded at a density of 3E4 cells/well. The inhibitors were resuspended in dimethyl sulfoxide (DMSO) prior to use and stored as recommended by the manufacturer as stock solutions (5 mM - $100 \mathrm{mM}$, varied depending dry weight and molecular weight of inhibitor received). Inhibitors were diluted to stated concentrations in cell culture media. Cells were pretreated with the target inhibitors before infection for $2 \mathrm{~h}$. Virus was diluted in media to appropriate MOI and placed on cells. The plate was incubated for 1 hour at $37^{\circ} \mathrm{C}, 5 \% \mathrm{CO} 2$ to allow the uptake of virus. Viral inoculum was removed, cells were gently washed three times with Dulbecco's phosphate-buffered saline (DPBS) without calcium and magnesium (Gibco 14190144) and conditioned media with the inhibitors was added back onto the cells. Cells were incubated at $37^{\circ} \mathrm{C}$ and supernatants were collected at indicated times post infection and stored at $-80^{\circ} \mathrm{C}$ until required for further analyses.

\section{Plaque assay.}

Plaque assays were performed using Vero cells grown to a cell number of 2 E5 cells/well in a 12well plate. Supernatants from infected cells were serially diluted in media and used to infect Vero cells for one hour. After infection a 1:1 overlay consisting of EMEM (without phenol red, supplemented with 10\% FBS, non-essential amino acids, $1 \mathrm{mM}$ sodium pyruvate, $2 \mathrm{mM}$ L-glutamine, $20 \mathrm{U} / \mathrm{ml}$ of penicillin and $20 \mu \mathrm{g} / \mathrm{ml}$ of streptomycin) and $0.6 \%$ agarose was added to each well. Plates were incubated at $37^{\circ} \mathrm{C}$ for 48 hours. Cells were fixed with $10 \%$ formaldehyde for 1 hour at room temperature. Formaldehyde was aspirated and the agarose overlay was removed. Cells were stained with crystal violet ( $1 \% \mathrm{CV}$ w/v in a $20 \%$ ethanol solution). Viral titer $(\mathrm{PFU} / \mathrm{mL})$ of SARS-CoV-2 was determined by counting the number of plaques.

\section{Cell viability assay.}

Cell viability was determined using the CellTiterGlo assay (Promega). Inhibitors were resuspended in DMSO and stored appropriately. Inhibitors were diluted to required concentrations in cell culture media. Vero cells were seeded at a density of 3E4 in white-walled 96-well plates. Inhibitors were overlaid on cells and incubated at $37^{\circ} \mathrm{C}, 5 \% \mathrm{CO} 2$ for 24 hours. CellTiterGlo substrate was used according to the manufacturer's instructions and luminescence was determined using a DTX 880 multimode detector (Beckman Coulter) with an integration time of 100ms/well. Cell viability was determined as percent cell viability normalized to untreated control.

\section{ACE2: Spike S1-Biotin (SARS-CoV-2) Inhibitor Screening Assay.}

Small molecules inhibitors were screened for ability to disrupt SARS-CoV-2 spike proteins binding to ACE2 receptors. ACE2: Spike S1-Biotin (SARS-CoV-2) Inhibitor Screening Assay Kit (BPS Bioscience, Cat\# 79945) was utilized per manufacturer's instructions. Plate was washed and blocked in between each addition step with provided wash buffer and blocking buffer. A nickel-coated plate was treated with his-tagged ACE2. Drugs were screened at a low concentration $(25 \mu \mathrm{M})$ and a high concentration $(100 \mu \mathrm{M})$. Inhibitor or DMSO was added to plate bring 
final dilution to indicated concentration. Plate was incubated for 1 hour at room temperature with shaking. SARS-CoV-2 spike S1-biotin was added to the plate. Plate was incubated for 1 hour at room temperature with shaking. Streptavidin-HRP is added to the plate. Plate was shaken for one hour at room temperature. HRP substrate was added to the plate Chemiluminescence was measured immediately using a DTX 880 multimode detector (Beckman Coulter) with an integration time of 1 second, and delay of 100ms.

\section{rVSV Pseudotyped SARS-CoV2 Spike Neutralization Assay.}

The vesicular stomatitis virus (rVSV) with deleted glycoprotein and replacement SARS-CoV-2 spike was acquired from IBT Bioservices. Vero cells were seed at a density of 6E4 in a black, 96-well tissue culture plate. Cells were maintained in EMEM supplemented with $10 \%$ fetal bovine serum (FBS) and 1x penicillin/streptomycin (P/S). Cells were incubated overnight $37^{\circ} \mathrm{C}$ and $5 \% \mathrm{CO}$. Virus was diluted per manufacture's protocol and incubated with drug at a final concentration of $5 \mu \mathrm{M}$ or DMSO control in unsupplemented media for one hour at room temperature. Media was removed from plate, replaced with treated or untreated virus. Plate was incubated for one hour at $37^{\circ} \mathrm{C}$. EMEM supplemented with $2 \% \mathrm{FBS}$ and $1 \mathrm{x} \mathrm{P} / \mathrm{S}$ was added to the wells and the plate was incubated overnight at $37^{\circ} \mathrm{C}$ and $5 \% \mathrm{CO} 2$. Cells were lysed with $1 \mathrm{X}$ Passive Lysis Buffer for 30 minutes while shaking. Firefly Luciferase Assay substrate was added to the plate. Plate was read immediately using a DTX 880 multimode detector (Beckman Coulter) with an integration time of 1 second.

\section{Transfection Methods.}

Vero cells were grown on 6-well plates and were transiently transfected with Lipofectamine 2000 (Invitrogen, 11668019) based on the manufacturer's protocols. In brief, prior to treatment, Lipofectamine 2000/siRNA complexes were prepared in reduced serum medium, OptiMEM (Gibco, 31985088), at the recommended ratio. Cells were treated for 6 hours before being replaced with EMEM complete medium.

\section{Microscopy analysis of cell-cell fusion.}

African green monkey kidney (Vero) cell lines (ATCC Cat\# CCL-81) were grown in Eagle's Minimum Essential Medium (EMEM) (Corning) supplemented with 10\% heat-inactivated fetal bovine serum (Gibco, USA). Cells were cultured at $37^{\circ} \mathrm{C}$ in a humidified incubator supplied with 5\% CO2. pTwist-EF1alpha-SARS-CoV-2-S-2xStrep (a gift from Nevan Krogan, Addgene plasmid \# 141382) was used to express SARS-CoV-2 S-protein. Lipofectamine 2000 (ThermoFisher) was used to transfect cells with the S-protein DNA (per manufactuer instructions). Maraviroc (Millipore Sigma, PZ0002) was dissolved in DMSO at a $1 \mathrm{mM}$ (stock). Before or following transfection, $5 \mu \mathrm{M}$ of Maraviroc solution was added to each well with complete EMEM medium.

Vero cells were transferred to $35 \mathrm{~mm}$ Glass bottom dishes (Cellvis, D35-20-1-N) 24 hours after transfection. Then cells were fixed with 4\% para-formaldehyde (Electron Microscopy Sciences, 15710) in PBS (Gibco, 14040182) 24 hours after attachment and then incubated with PBS. 
Thereafter, cells were mounted using Prolong Diamond Antifade Kit (Invitrogen, P36970) according to manufacturer's instructions. Anti-Lamin A (abcam, ab26300) with secondary donkey anti-Mouse IgG (ThermoFisher, A-31571) and Hoechst 33342 (ThermoFisher, H3570) were used to identify nuclei. StrepMAB-Classic DY-488 conjugate (IBA Lifesciences, 2-1563-050) was used to label the Strep-tag II labeled S-protein. Actin-stain 555 phalloidin (Cytoskeleton, PHDH1-A) was used to label the actin cytoskeleton. Cells were imaged using a Zeiss LSM 710 Laser Scanning Microscope. Images were analyzed by Fiji (U.S. National Institutes of Health, Bethesda, MD). Total nuclei and S-protein transfected nuclei were counted using Cell Counter plugin.

\section{Simulation modeling.}

To simulate Maraviroc treatments, we used an open source, community-developed simulator (Version 3.2) for SARS-CoV-2 infection dynamics in tissue that currently includes diffusive viral spread through the tissue, ACE2 receptor dynamics, intracellular viral replication, single-cell viral and antiviral responses, and immune response (Getz et al., 2020). The simulator uses an agent-based modeling approach (PhysiCell (Ghaffarizadeh et al., 2018)) to simulate individual epithelial and immune cells. After adapting to the in vitro experimental conditions (no immune components), we added a pharmacodynamics model to each simulated cell. We also assumed that ACE2 binding and endocytosis rates may be reduced in infected cells. See Getz et al. (2020) for full details on the ACE2 receptor binding and trafficking, viral replication and exocytosis, and infected cell death response. See Fig. 8A-B for the model overview.

To model the single-cell pharmacodynamics, we first modeled the rate of drug internalization as diffusion through the cell membrane, allowing us to track the accumulated total drug in each cell (Equation 1). Similarly to Aljayyoussi et al. (2017) and Aljayyoussi et al. (2019), we model the total effect $E$ for each cell based on the internal drug concentration $\rho_{\text {intra }}$ using a Hill function (Equation 2). The effect is then used to modulate a vector $\boldsymbol{r}$ of cell parameters from their untreated rates $\left(\boldsymbol{r}_{0}\right)$ to their rates at maximum drug efficacy at $\left(\boldsymbol{r}_{\max }\right)$ when $E=E_{\max }$. (Equation 3 ). In this investigation, $\boldsymbol{r}$ includes the viral endocytosis and export rates. In addition, we can model the impact of partial drug efficacy by multiplying the effect by a sensitivity $S$, where $S=0$ denotes a drug with no efficacy (e.g., it fails to bind its target, or binding its target does not affect cell phenotype), and $S=100 \%$ is an idea drug that fully binds and inhibits its target.

$$
\begin{gathered}
\frac{d n}{d t}=r_{I} \cdot\left(\rho_{\text {extra }}-\rho_{\text {intra }}\right) \cdot V_{\text {cell }} \\
E=E_{\text {max }} \cdot \frac{\rho_{\text {intra }}^{n}}{\mathrm{EC}_{50}^{n}+\rho_{\text {intra }}^{n}} \\
\boldsymbol{r}=\boldsymbol{r}_{0}+\left(\boldsymbol{r}_{\max }-\boldsymbol{r}_{0}\right) \cdot \frac{E}{E_{\max }} \cdot S
\end{gathered}
$$

Here, $r_{I}$ is drug internalization rate, $\rho_{\text {extra }}$ and $\rho_{\text {intra }}$ are the extracellular and intracellular drug concentrations, $V_{\text {cell }}$ is cell volume. $E_{\max }$ is maximum drug effect (typically normalized to 1 ), $\mathrm{EC}_{50}$ is the drug dose that gives $50 \%$ of maximum effect, and $n$ is Hill power (Goutelle et al., 2008). 
Full simulation source code is available as open source under the BSD license; see Appendix A. We used xml2jupyter (Heiland et al., 2019) to create a cloud-hosted version of this model; interested readers can interactively run the model in a web browser at https://nanohub.org/tools/virion2pbpd ). See Figure 8C-D.

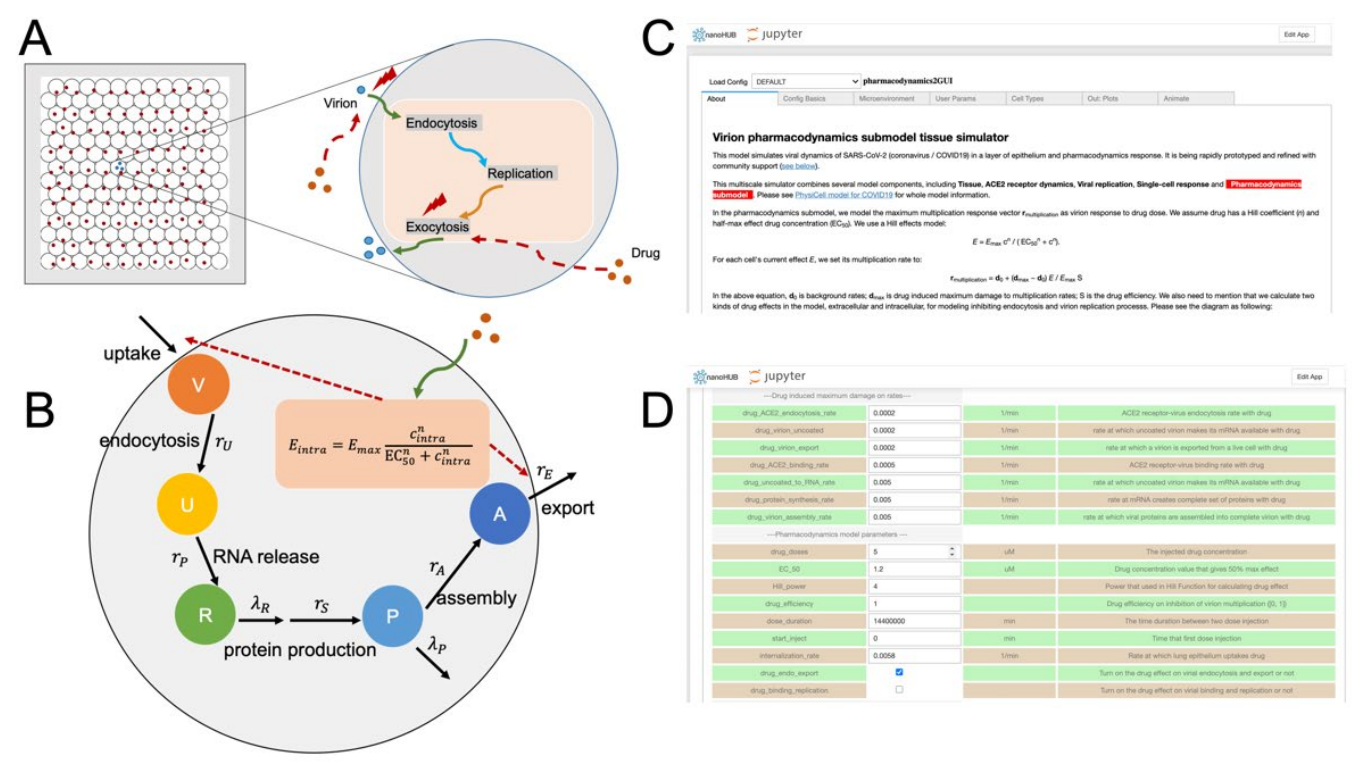

Figure 8: (A-B): Simulation model schematics. (C-D): Typical user interface for the webhosted simulation model.

\section{Simulation model calibration.}

We began with the default parameter values from Getz et al. (2020) and adjusted to match viral titer measurements at 16 hours under control conditions and a $5 \mu \mathrm{M}$ dose of Maravirac. First, we adjusted the receptor dynamics parameters (ACE2 binding rate, ACE2 endocytosis rate, ACE2 cargo release rate and ACE2 recycling rate), viral replication parameters (virion uncoating rate, RNA preparation rate, viral protein synthesis rate, and virion assembly rate) and export rate to match the viral titer in untreated Vero cells at 16 hours, assuming that 3000 cells are initially infected.

Prior experiments show that peak cytoplasmic drug concentrations are reached in about 3.1 hours (300mg dose) (Carter and Keating, 2007). To match this drug uptake time scale, we set the internalization $r_{I}$ at $0.0116 \mu \mathrm{M} \cdot \mu \mathrm{m}^{3} / \mathrm{min}$ (1 hour to reach half maximum). We initially approximated $\mathrm{EC}_{50}$ as $2.5 \mu \mathrm{M}$ (half of experimental dose) and the Hill power $n$ as 1 in the first pass. In order to match with the viral titer at 16 hours with a dose of $5 \mu \mathrm{M}$, we varied $\mathrm{EC}_{50}$ and the Hill power until the full dynamical model yielded a viral titer that matched experimental measurements. The resulting parameters were $\mathrm{EC}_{50}=2.7 \mu \mathrm{M}$ and $n=2.8$. See Goutelle et al. (2008) for more details on common pharmacodynamic forms.

\section{Computing.}


We used a total of 12 parameters sets. Because the simulation model is stochastic, we ran 10 simulation replicates for each parameter set. All simulation images are chosen from one representative replicate, and all aggregate dynamical curves (e.g., viral titer versus time) are reported as the mean over all 10 simulation replicates. The simulations were performed on the Big Red 3 supercomputer at Indiana University. Each simulation was run on a single node using 32 threads. All jobs (120 runs) were submitted as a batch. Each simulation was completed in approximately 35 minutes, and with a total wall time of approximately of 3 hours.

\section{Statistical analysis.}

Statistical analyses were performed using the software Prism 5 (Graph Pad). Data are presented as mean \pm standard deviation (SD) after analysis with unpaired, two-tailed t-test or one-way ANOVA. Differences in statistical significance are indicated with asterisks: ${ }^{*} \mathrm{p}<0.05 ; * * \mathrm{p}<0.01$; $* * * \mathrm{p}<0.001 ; * * * * \mathrm{p}<0.0001$.

\section{Appendix A: Simulate source code}

The simulation source code is available at:

GitHub repository: $\quad$ https://github.com/pc4covid19/pharmacodynamics-submodel

Zenodo archive: $\quad$ https://zenodo.org/badge/latestdoi/282789470

The nanoHUB web version of the model can be accessed at:

nanoHUB DOI: $\quad$ http://dx.doi.org/doi:10.21981/CCFV-ES15

\section{References}

Ahmed, S.F., Quadeer, A.A., and McKay, M.R. (2020). Preliminary Identification of Potential Vaccine Targets for the COVID-19 Coronavirus (SARS-CoV-2) Based on SARS-CoV Immunological Studies. Viruses 12, 254.

Aljayyoussi, G., Donnellan, S., Ward, S.A., and Biagini, G.A. (2019). Intracellular PD Modelling (PDi) for the Prediction of Clinical Activity of Increased Rifampicin Dosing. Pharmaceutics 11 .

Aljayyoussi, G., Jenkins, V.A., Sharma, R., Ardrey, A., Donnellan, S., Ward, S.A., and Biagini, G.A. (2017). Pharmacokinetic-Pharmacodynamic modelling of intracellular Mycobacterium tuberculosis growth and kill rates is predictive of clinical treatment duration. Scientific Reports 7. 
Astuti, I., and Ysrafil (2020). Severe Acute Respiratory Syndrome Coronavirus 2 (SARS-CoV2): An overview of viral structure and host response. Diabetes Metab Syndr 14, 407-412.

Banerjee, A., Kulcsar, K., Misra, V., Frieman, M., and Mossman, K. (2019). Bats and Coronaviruses. Viruses 11, 41 .

Bansal, M. (2020). Cardiovascular disease and COVID-19. Diabetes Metab Syndr 14, 247-250.

Camm, J., Hla, T., Bakshi, R., and Brinkmann, V. (2014). Cardiac and vascular effects of fingolimod: Mechanistic basis and clinical implications. American Heart Journal 168, 632-644.

Carter, N.J., and Keating, G.M. (2007). Maraviroc. Drugs 67, 2277-2288.

Ciceri, F., Beretta, L., Scandroglio, A.M., Colombo, S., Landoni, G., Ruggeri, A., Peccatori, J., D'Angelo, A., De Cobelli, F., Rovere-Querini, P., et al. (2020). Microvascular COVID-19 lung vessels obstructive thromboinflammatory syndrome (MicroCLOTS): an atypical acute respiratory distress syndrome working hypothesis. Crit Care Resusc 22, 95-97.

De Wit, E., Van Doremalen, N., Falzarano, D., and Munster, V.J. (2016). SARS and MERS: recent insights into emerging coronaviruses. Nature Reviews Microbiology 14, 523-534.

Dong, E., Du, H., and Gardner, L. (2020). An interactive web-based dashboard to track COVID19 in real time. The Lancet Infectious Diseases 20, 533-534.

Fanelli, V., Vlachou, A., Ghannadian, S., Simonetti, U., Slutsky, A.S., and Zhang, H. (2013). Acute respiratory distress syndrome: new definition, current and future therapeutic options. $\mathrm{J}$ Thorac Dis 5, 326-334.

Fehr, A.R., and Perlman, S. (2015). Coronaviruses: an overview of their replication and pathogenesis. Methods Mol Biol 1282, 1-23.

Ge, X.-Y., Li, J.-L., Yang, X.-L., Chmura, A.A., Zhu, G., Epstein, J.H., Mazet, J.K., Hu, B., Zhang, W., Peng, C., et al. (2013). Isolation and characterization of a bat SARS-like coronavirus that uses the ACE2 receptor. Nature 503, 535-538.

Getz, M., Wang, Y., An, G., Becker, A., Cockrell, C., Collier, N., Craig, M., Davis, C.L., Faeder, J., Versypt, A.N.F., et al. (2020).

Ghaffarizadeh, A., Heiland, R., Friedman, S.H., Mumenthaler, S.M., and Macklin, P. (2018). PhysiCell: An open source physics-based cell simulator for 3-D multicellular systems. PLOS Computational Biology 14.

Glowacka, I., Bertram, S., Müller, M.A., Allen, P., Soilleux, E., Pfefferle, S., Steffen, I., Tsegaye, T.S., He, Y., Gnirss, K., et al. (2011). Evidence that TMPRSS2 activates the severe acute respiratory syndrome coronavirus spike protein for membrane fusion and reduces viral control by the humoral immune response. J Virol 85, 4122-4134. 
Goutelle, S., Maurin, M., Rougier, F., Barbaut, X., Bourguignon, L., Ducher, M., and Maire, P. (2008). The Hill equation: a review of its capabilities in pharmacological modelling.

Fundamental \& Clinical Pharmacology 22, 633-648.

Heiland, R., Mishler, D., Zhang, T., Bower, E., and Macklin, P. (2019). xml2jupyter: Mapping parameters between XML and Jupyter widgets. Journal of Open Source Software 4.

Kam, Y.-W., Okumura, Y., Kido, H., Ng, L.F.P., Bruzzone, R., and Altmeyer, R. (2009). Cleavage of the SARS coronavirus spike glycoprotein by airway proteases enhances virus entry into human bronchial epithelial cells in vitro. PloS one 4, e7870-e7870.

Kelleni, M.T. (2020). Nitazoxanide/azithromycin combination for COVID-19: A suggested new protocol for early management. Pharmacol Res 157, 104874-104874.

Liu, X., and Zhang, S. (2020). COVID-19: Face masks and human-to-human transmission. Influenza and Other Respiratory Viruses 14, 472-473.

Lontok, E., Corse, E., and Machamer, C.E. (2004). Intracellular targeting signals contribute to localization of coronavirus spike proteins near the virus assembly site. J Virol 78, 5913-5922.

Mackay, I.M., and Arden, K.E. (2015). MERS coronavirus: diagnostics, epidemiology and transmission. Virol J 12.

Madu, I.G., Roth, S.L., Belouzard, S., and Whittaker, G.R. (2009). Characterization of a highly conserved domain within the severe acute respiratory syndrome coronavirus spike protein S2 domain with characteristics of a viral fusion peptide. J Virol 83, 7411-7421.

Mahfouz, N., Tahtouh, R., Alaaeddine, N., El Hajj, J., Sarkis, R., Hachem, R., Raad, I., and Hilal, G. (2017). Gastrointestinal cancer cells treatment with bevacizumab activates a VEGF autoregulatory mechanism involving telomerase catalytic subunit hTERT via PI3K-AKT, HIF$1 \alpha$ and VEGF receptors. PLOS ONE 12, e0179202.

Martins-Filho, P.R., Barreto-Alves, J.A., and Fakhouri, R. (2020). Potential role for nitazoxanide in treating SARS-CoV-2 infection. American Journal of Physiology-Lung Cellular and Molecular Physiology 319, L35-L36.

Menachery, V.D., Yount, B.L., Debbink, K., Agnihothram, S., Gralinski, L.E., Plante, J.A., Graham, R.L., Scobey, T., Ge, X.-Y., Donaldson, E.F., et al. (2015). A SARS-like cluster of circulating bat coronaviruses shows potential for human emergence. Nature Medicine 21, 15081513.

Nishiura, H., Linton, N.M., and Akhmetzhanov, A.R. (2020). Initial Cluster of Novel Coronavirus (2019-nCoV) Infections in Wuhan, China Is Consistent with Substantial Human-toHuman Transmission. J Clin Med 9, 488.

Ou, X., Liu, Y., Lei, X., Li, P., Mi, D., Ren, L., Guo, L., Guo, R., Chen, T., Hu, J., et al. (2020). Characterization of spike glycoprotein of SARS-CoV-2 on virus entry and its immune crossreactivity with SARS-CoV. Nature communications 11, 1620-1620. 
Papachristos, A., Kemos, P., Katsila, T., Panoilia, E., Patrinos, G.P., Kalofonos, H., and Sivolapenko, G.B. (2019). VEGF-A and ICAM-1 Gene Polymorphisms as Predictors of Clinical Outcome to First-Line Bevacizumab-Based Treatment in Metastatic Colorectal Cancer. Int J Mol Sci 20, 5791.

Pul, R., Osmanovic, A., Schmalstieg, H., Pielen, A., Pars, K., Schwenkenbecher, P., Sühs, K.W., Yildiz, Ö., Frank, B., Stangel, M., et al. (2016). Fingolimod Associated Bilateral Cystoid Macular Edema-Wait and See? Int J Mol Sci 17, 2106.

Ramirez, J.A.C., Ruth; Cavallazzi,, Rodrigo; Beavin, L.R., Anupama; Burns, Mark V.; Mahmood, Kamran; Aden, Darmaan; Prabhu, A.B., Dawn;, Furmanek, S.W., Leslie; Palmer, Kenneth E.; Tella, M.G., Connor; Arnold, Forest W. and the Center of Excellence, (CERID), f.R.i.I.D., and (2020)., C.S.G. (2020). Community-Acquired Pneumonia due to Endemic Human

Coronaviruses compared to 2019 Novel Coronavirus: A Review. University of Louisville Journal of Respiratory Infections 4, 1-9.

Rossignol, J.F. (2016). Nitazoxanide, a new drug candidate for the treatment of Middle East respiratory syndrome coronavirus. J Infect Public Health 9, 227-230.

Simmons, G., Bertram, S., Glowacka, I., Steffen, I., Chaipan, C., Agudelo, J., Lu, K., Rennekamp, A.J., Hofmann, H., Bates, P., et al. (2011). Different host cell proteases activate the SARS-coronavirus spike-protein for cell-cell and virus-cell fusion. Virology 413, 265-274.

Sisk, J.M., Frieman, M.B., and Machamer, C.E. (2018). Coronavirus S protein-induced fusion is blocked prior to hemifusion by Abl kinase inhibitors. J Gen Virol 99, 619-630.

Song, R., Han, B., Song, M., Wang, L., Conlon, C.P., Dong, T., Tian, D., Zhang, W., Chen, Z., Zhang, F., et al. (2020). Clinical and epidemiological features of COVID-19 family clusters in Beijing, China. Journal of Infection 81, e26-e30.

Stadler, K., Masignani, V., Eickmann, M., Becker, S., Abrignani, S., Klenk, H.-D., and Rappuoli, R. (2003). SARS - beginning to understand a new virus. Nature Reviews Microbiology 1, 209-218.

Tang, T., Bidon, M., Jaimes, J.A., Whittaker, G.R., and Daniel, S. (2020). Coronavirus membrane fusion mechanism offers a potential target for antiviral development. Antiviral Res $178,104792-104792$.

Trejo-Gabriel-Galán, J.M. (2020). Stroke as a complication and prognostic factor of COVID-19. Neurologia 35, 318-322.

Wang, L.-F., Shi, Z., Zhang, S., Field, H., Daszak, P., and Eaton, B.T. (2006). Review of bats and SARS. Emerg Infect Dis 12, 1834-1840.

Weiss, S.R., and Leibowitz, J.L. (2011). Coronavirus pathogenesis. Adv Virus Res 81, 85-164. 
Xia, S., Liu, M., Wang, C., Xu, W., Lan, Q., Feng, S., Qi, F., Bao, L., Du, L., Liu, S., et al. (2020). Inhibition of SARS-CoV-2 (previously 2019-nCoV) infection by a highly potent pancoronavirus fusion inhibitor targeting its spike protein that harbors a high capacity to mediate membrane fusion. Cell Research 30, 343-355.

Ye, Q., Wang, B., and Mao, J. (2020). The pathogenesis and treatment of the 'Cytokine Storm' in COVID-19. Journal of Infection 80, 607-613.

Zhang, X.J., Qin, J.J., Cheng, X., Shen, L., Zhao, Y.C., Yuan, Y., Lei, F., Chen, M.M., Yang, H., Bai, L., et al. (2020). In-Hospital Use of Statins Is Associated with a Reduced Risk of Mortality among Individuals with COVID-19. Cell Metab 32, 176-187.e174.

Zhong, N.-S., and Wong, G.W.K. (2004). Epidemiology of severe acute respiratory syndrome (SARS): adults and children. Paediatric Respiratory Reviews 5, 270-274.

Zhou, P., Yang, X.-L., Wang, X.-G., Hu, B., Zhang, L., Zhang, W., Si, H.-R., Zhu, Y., Li, B., Huang, C.-L., et al. (2020). A pneumonia outbreak associated with a new coronavirus of probable bat origin. Nature. 
A.

\begin{tabular}{|c|c|c|}
\hline 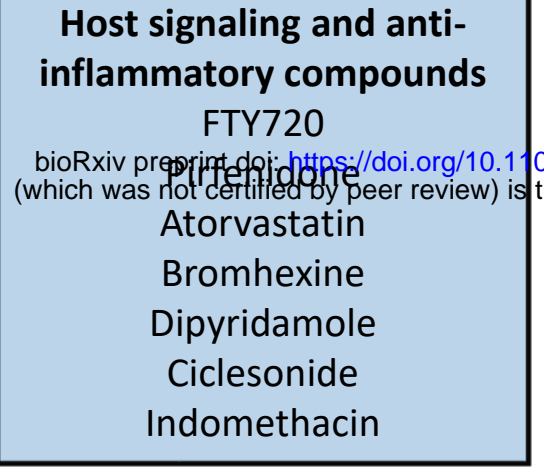 & 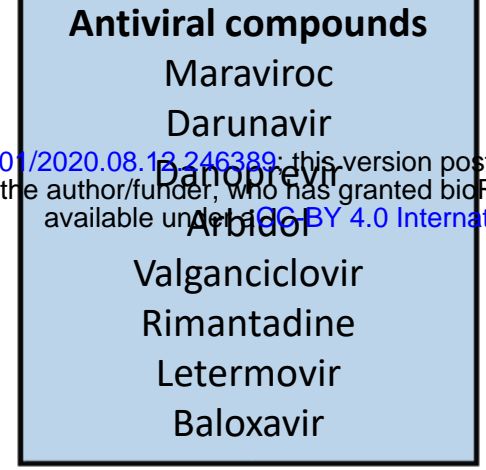 & 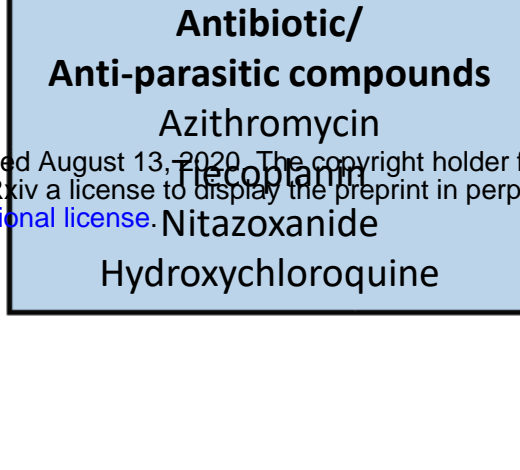 \\
\hline
\end{tabular}

B.

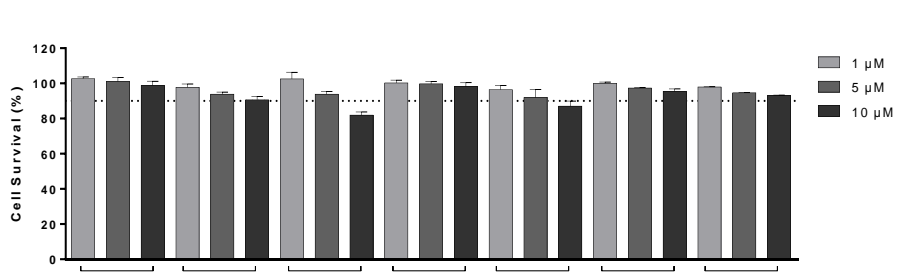

12

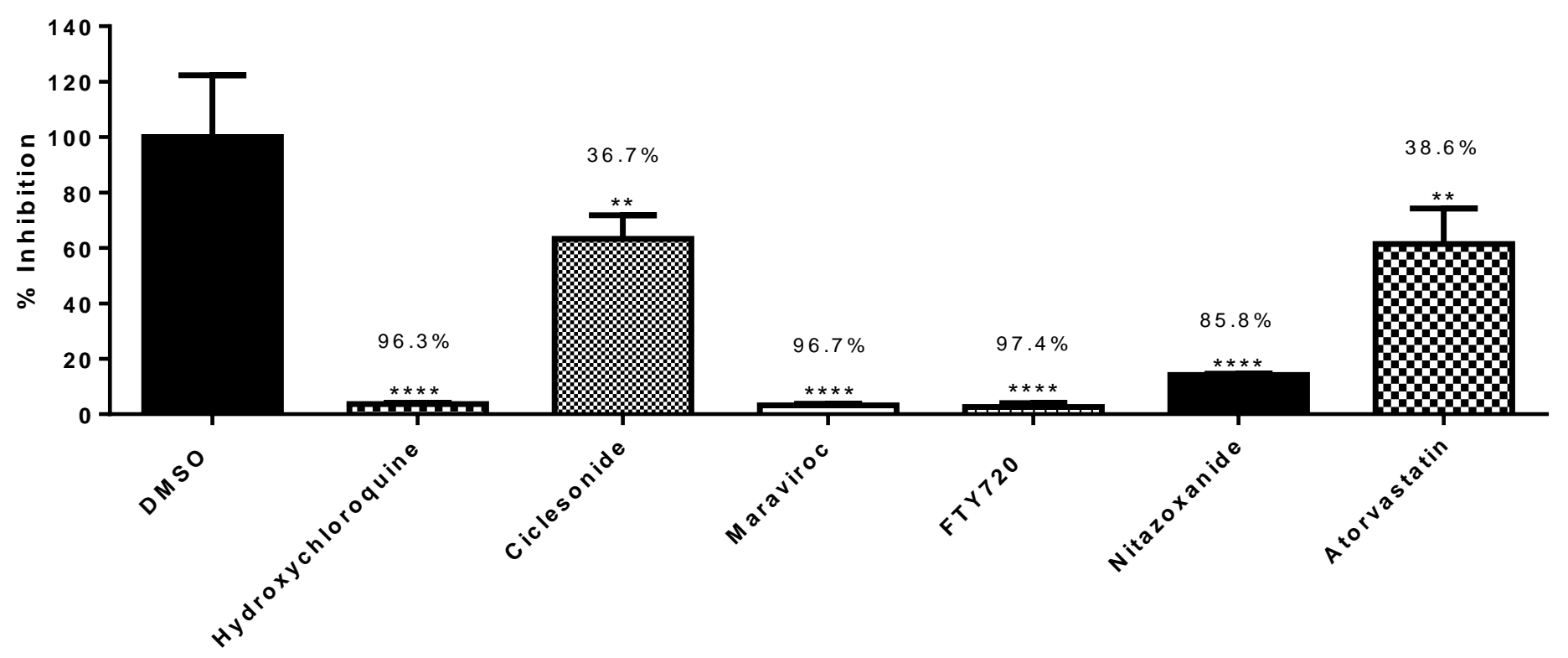

Risner et al., Figure 1 
A.

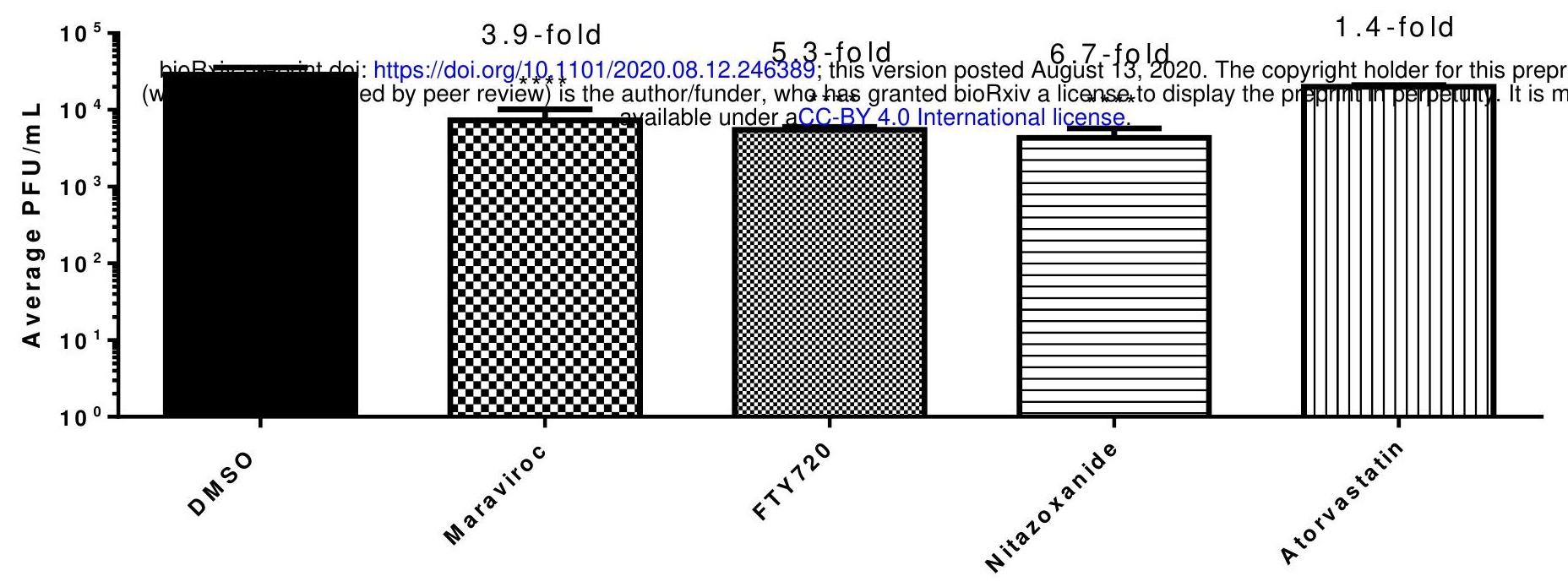

B.

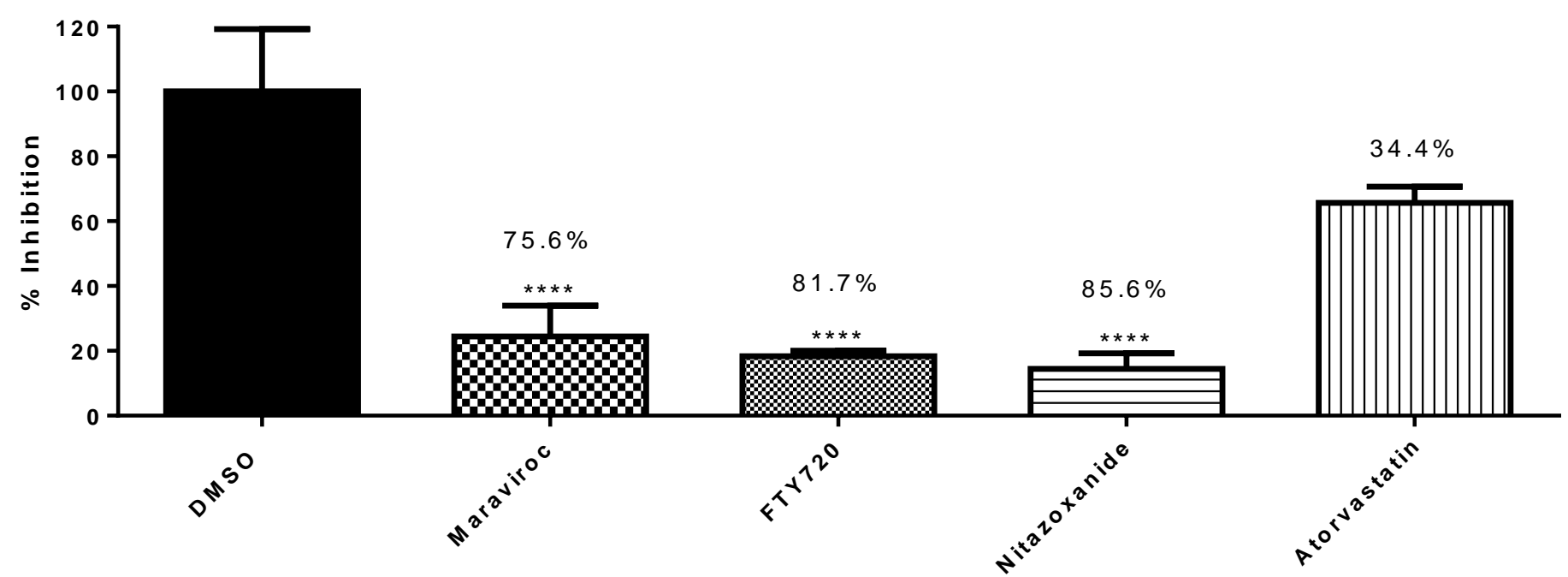

Risner et al., Figure 3 
Figure 5.

A.

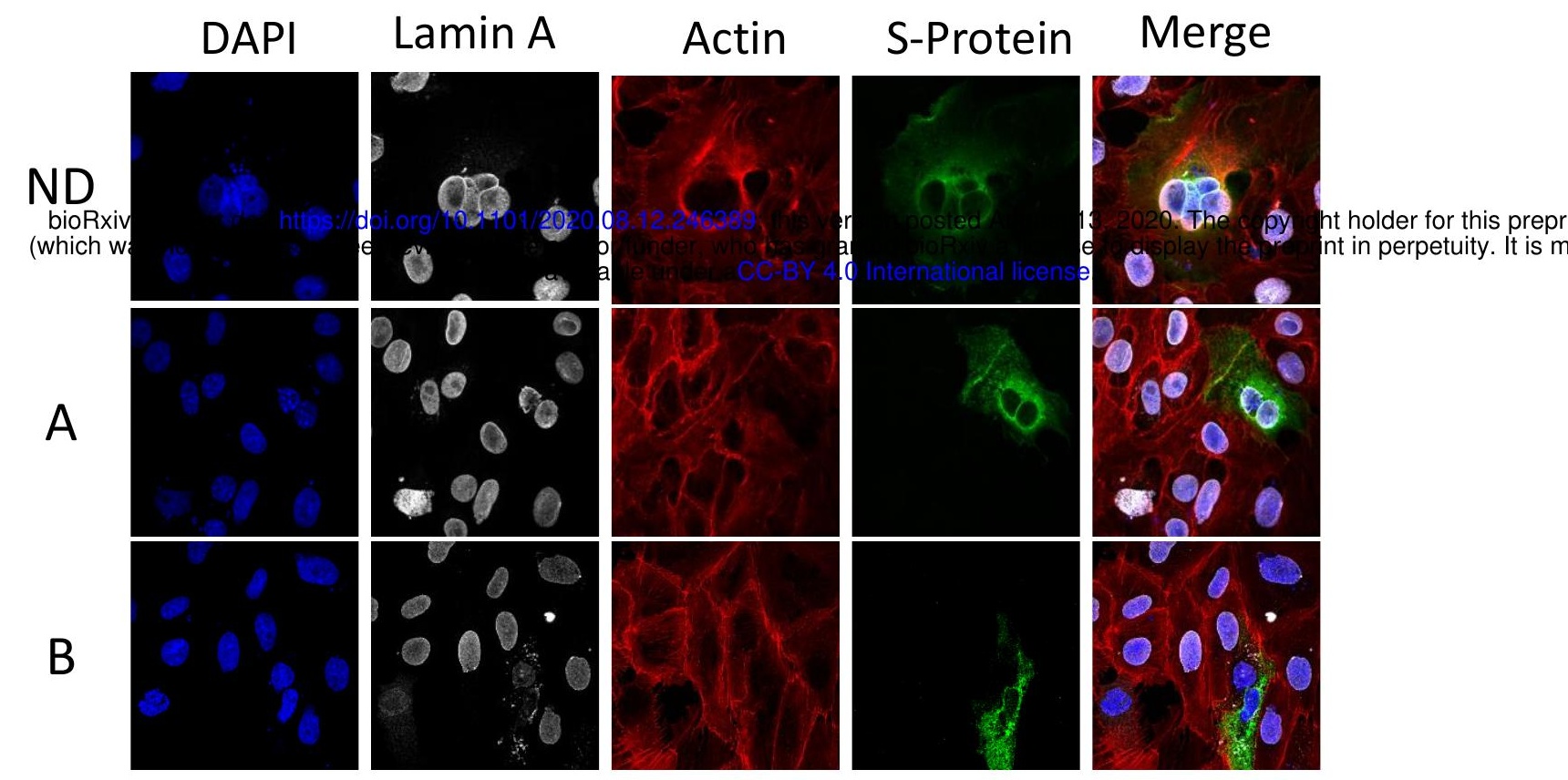

B.

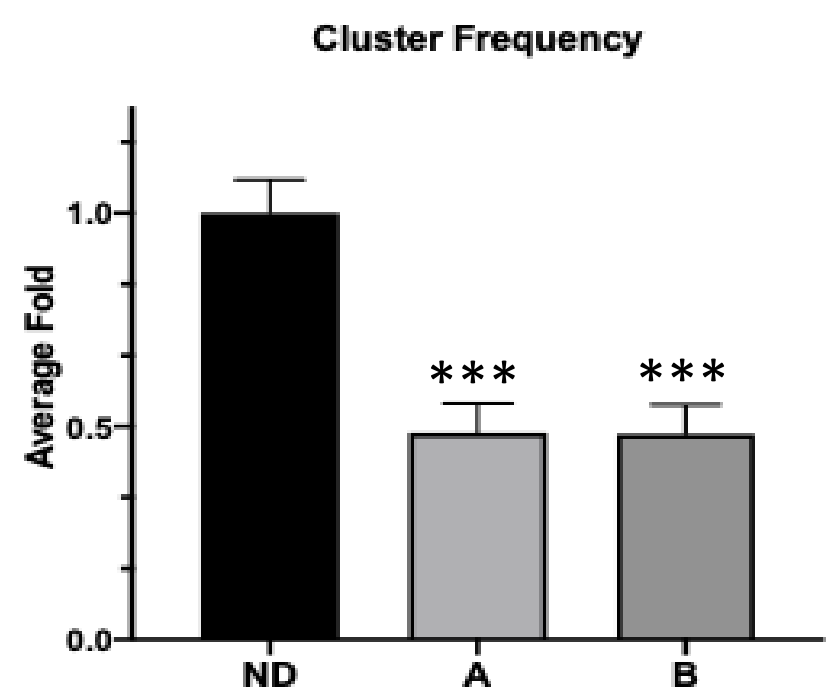

C.

Number of Nuclei Per Cluster

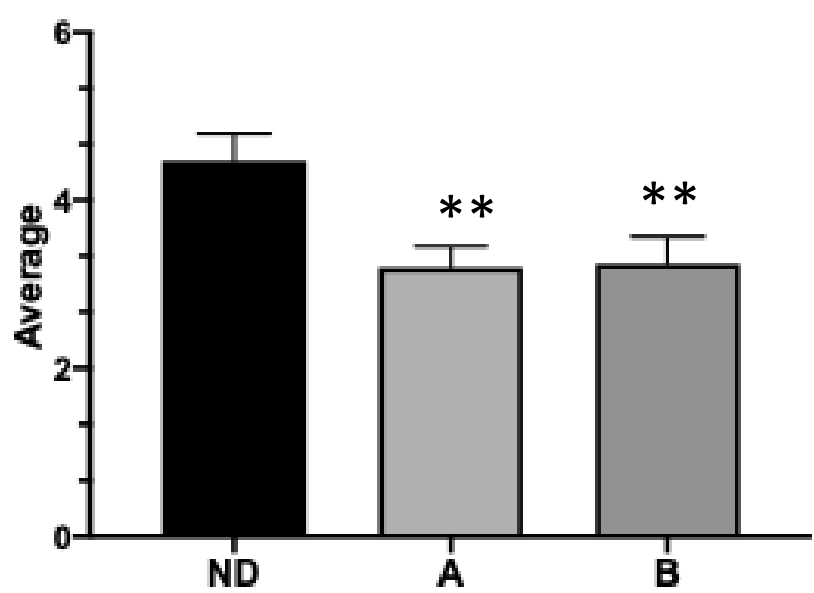

Risner et al., Figure 5 

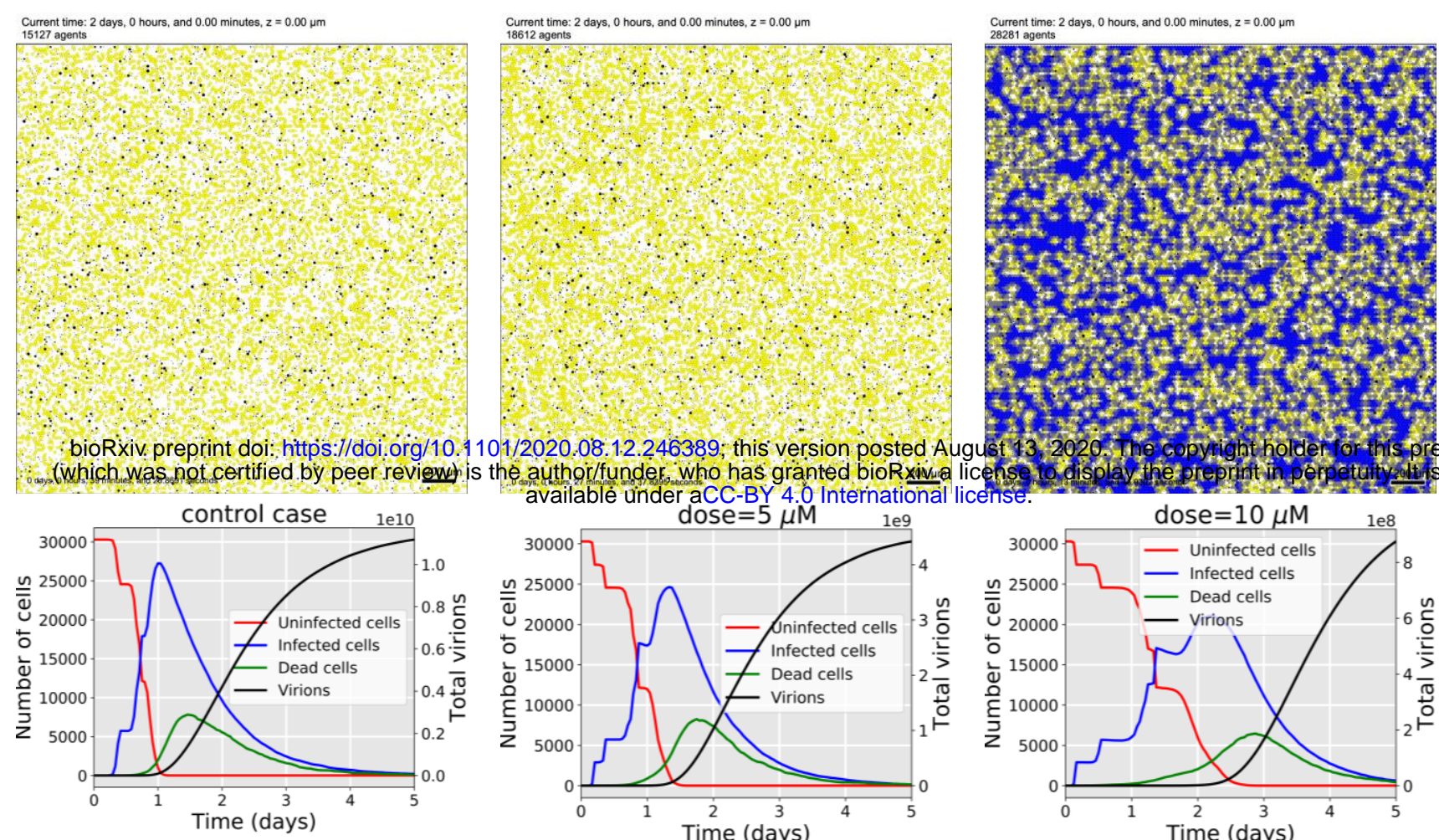

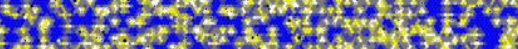
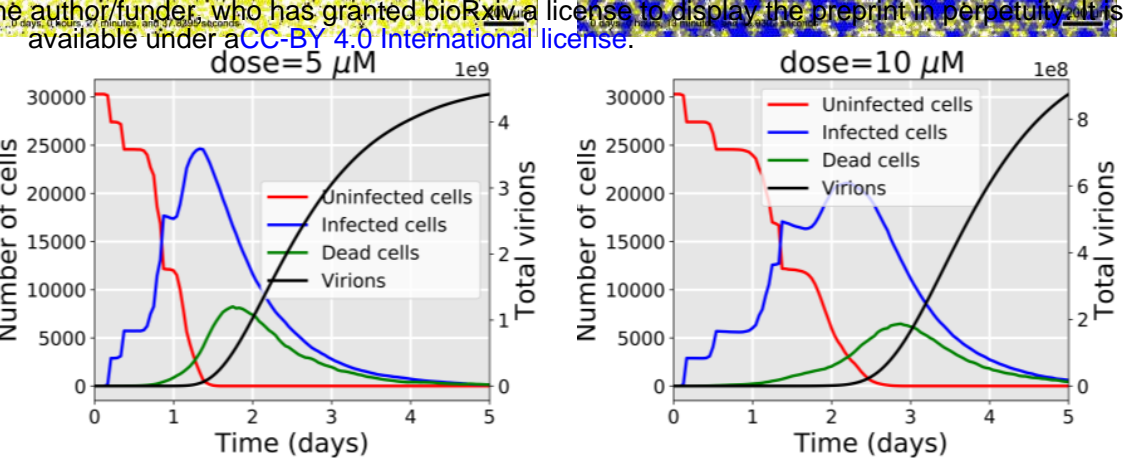

B

$\mathrm{Oh}$

$8 \mathrm{~h}$

$24 \mathrm{~h}$
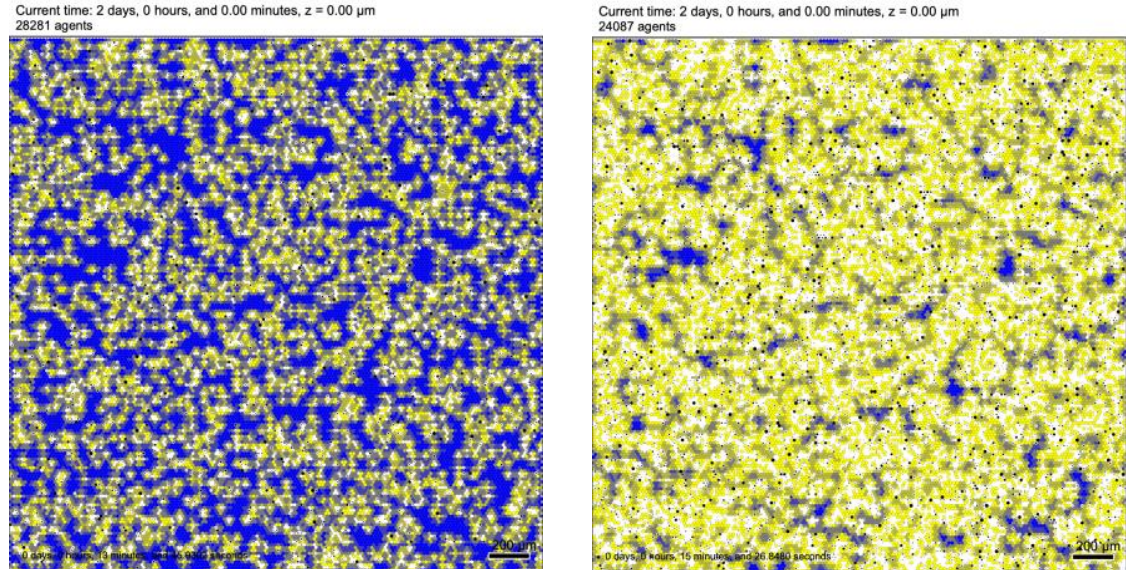

Current time: 2 days, 0 hours, and 0.00 minutes, $z=0.00 \mathrm{\mu m}$
12034 agents
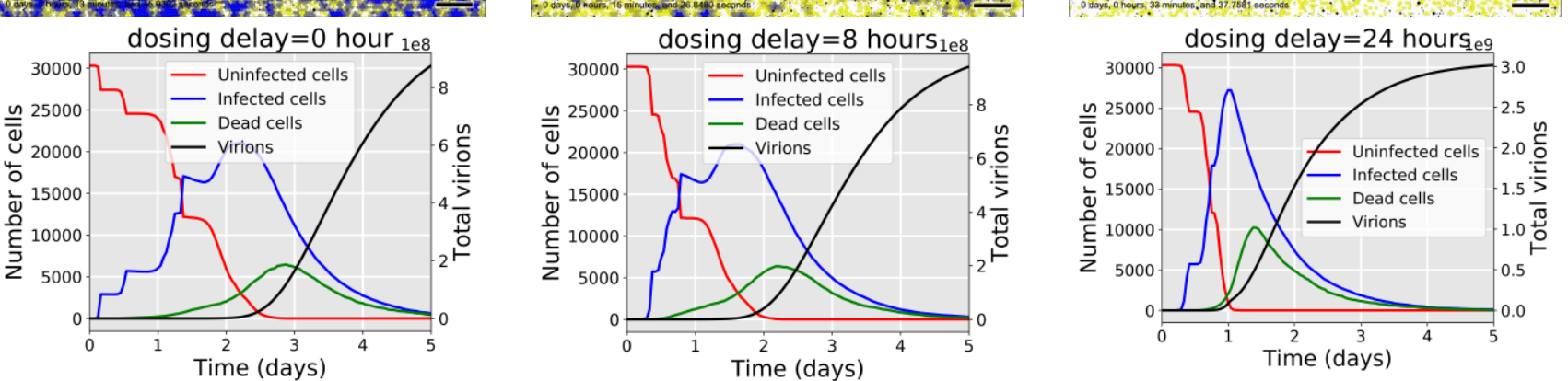

C

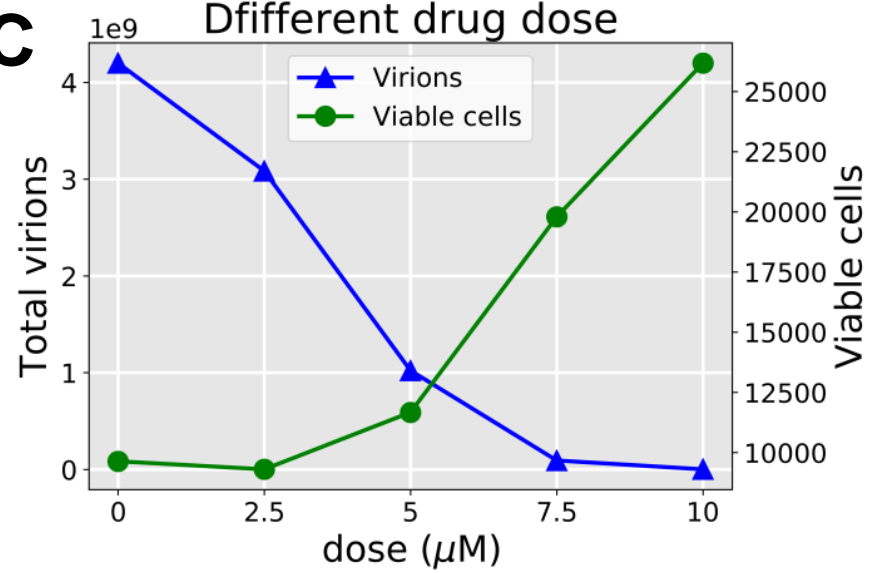

D 1 e9 Dfifferent dosing delay

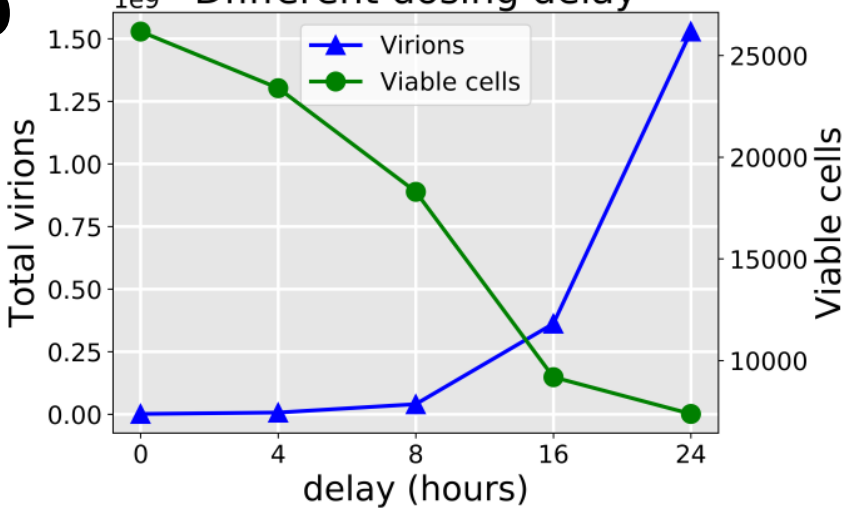




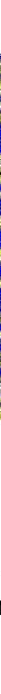

Fig S3: Impact of decreasing Maraviroc sensitivity. Top row: As the cells' maximum inhibitory response to a $10 \mu \mathrm{M}$ dose of Maraviroc is increased from $0 \%$ inhibition of endocytosis and exocytosis to $100 \%$ inhibition, the number of uninfected cells (deep blue) at 48 hours increases, while the number of infected cells with high viral load (bright yellow cells) and dead cells (black) decreases. Bottom row: As sensitivity is increased, the infection's progression is slowed, as seen by delayed increases in viral titer, infected cells, and dead cells. The final viral titer steadily decreases with increasing dose.

$0 \mathrm{~h}$

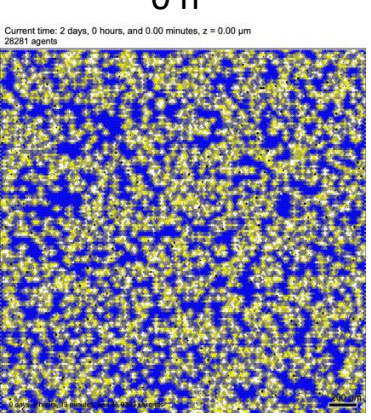

dosing delay $=0$ hour

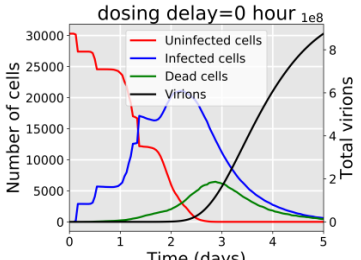

$4 \mathrm{~h}$

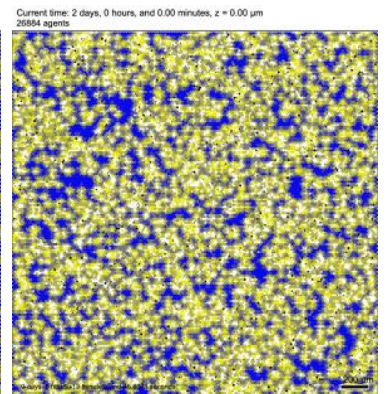

dosing delay $=4$ hours

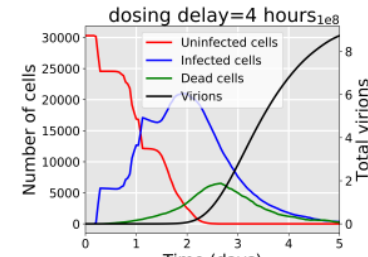

$8 \mathrm{~h}$

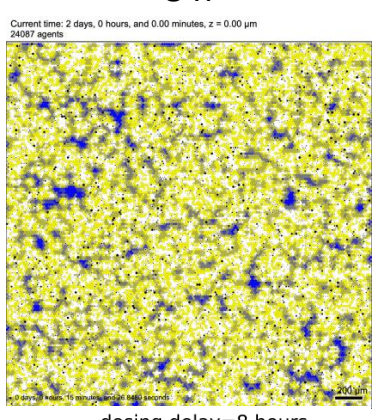

$16 \mathrm{~h}$

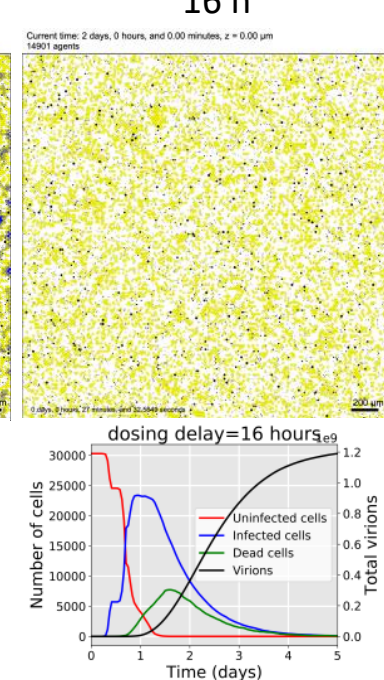

$24 \mathrm{~h}$
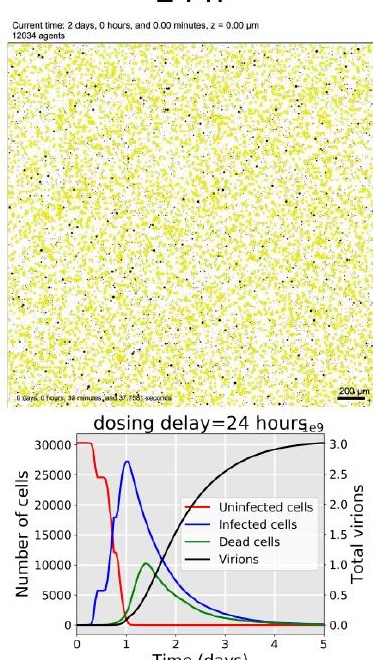

Fig S4: Impact of delaying Maraviroc treatment. Top row: As the $10 \mu \mathrm{M}$ Maraviroc treatment is delayed by longer times, the number of uninfected cells (deep blue) at 48 hours increases, while the number of infected cells with high viral load (bright yellow cells) and dead cells (black) decreases. Bottom row: As the treatment delay is increased, the infection's progression is accelerated, as seen by earlier increases in viral titer, infected cells, and dead cells. The final viral titer steadily increases with increasing treatment delay. 